TRANSACTIONS OF THE

AMERICAN MATHEMATICAL SOCIETY

Volume 350, Number 1, January 1998, Pages 185-211

S 0002-9947(98)02007-8

\title{
NEW SUBFACTORS FROM BRAID GROUP REPRESENTATIONS
}

\author{
JULIANA ERLIJMAN
}

\begin{abstract}
This paper is about the construction of new examples of pairs of subfactors of the hyperfinite $\mathrm{II}_{1}$ factor, and the computation of their indices and relative commutants. The construction is done in general by considering unitary braid representations with certain properties that are satisfied in natural examples. We compute the indices explicitly for the particular cases in which the braid representations are obtained in connection with representation theory of Lie algebras of types A,B,C,D.
\end{abstract}

\section{INTRODUCTION}

Jones defined in $[\mathrm{J}]$ the index $[M: N]$ for a pair of $\mathrm{II}_{1}$ factors $N \subseteq M$ as the coupling constant of $N$ in its representation on $L^{2}(M, t r)$. This is an invariant of subfactors up to conjugacy by automorphisms. He showed that the values for the index are either greater than 4 or lie in the set $\left\{4 \cos ^{2}(\pi / l)\right.$ for $\left.l \geq 3, l \in \mathbb{N}\right\}$. Another invariant is the relative commutant or centralizer $N^{\prime} \cap M$. The subfactors with index less than 4 have always trivial relative commutant (called irreducible subfactors).

In this paper we construct a new family of examples of subfactors of the hyperfinite $\mathrm{II}_{1}$ factor, and compute their indices and (first) relative commutants. By 'reducing' the pairs by minimal projections in the relative commutant we obtain irreducible subfactors whose indices we also compute.

This construction is done in general by considering unitary representations $\rho$ of the infinite braid group whose restrictions to finite braid groups generate finite dimensional $\mathrm{C}^{*}$-algebras (such representations are called locally finite dimensional). We require certain auxiliary conditions on the representations, such as the existence of a positive Markov trace factoring through the representation, which are satisfied in natural examples. One can extend such a representation $\rho$ to the infinite twosided braid group with generators $\sigma_{i}, i \in \mathbb{Z}$. Then, for each non-negative integer $m$ we consider the pair of von Neumann algebras generated by $\left\{\pi_{t r}\left(g_{i}\right): i \in\right.$ $\mathbb{Z} \backslash\{0, \ldots m\}\}$ and by $\left\{\pi_{t r}\left(g_{i}\right): i \in \mathbb{Z}\right\}$ in the trace representation, where $g_{i}=\rho\left(\sigma_{i}\right)$. Under our assumptions, this is a pair of factors, and the relative commutant is generated by $\left\{g_{1}, \ldots, g_{m-1}\right\}$.

This construction is a 'two-sided' version of a construction due to Wenzl, [W-1], [W-2], [W-3]. For $m=0$ (that is, when one braid generator, $g_{0}$, is ommited) the index $d^{2}$ is the square of the euclidean norm of a (normalized) weight vector for the

Received by the editors January 24, 1996.

1991 Mathematics Subject Classification. Primary 46L37.

(C)1998 American Mathematical Society 
trace on $\rho\left(\mathbb{C B}_{n}\right)$, for large $n$. For $m \geq 1$, the index is given by $d^{2} W^{m}$, where $W$ is the index for the 'one-sided' pair, $\left\{\pi_{t r}\left(g_{i}\right): i \geq 2\right\}^{\prime \prime} \subseteq\left\{\pi_{t r}\left(g_{i}\right): i \geq 1\right\}^{\prime \prime}$.

We compute the indices explicitly for the particular cases in which the braid representations factor through the Hecke algebra $H_{\infty}(q)$ when $q$ is a root of unity (type A case, $[\mathrm{W}-1]$ ) or through the Birman-Murakami-Wenzl algebra $C_{\infty}(r, q)$ for special values of $r$ and $q$ (types B,C and D cases, [W-2]). Our type A subfactors include subfactors obtained by $\mathrm{M}$. Choda (type $\mathrm{A}_{1},[\mathrm{Ch}]$ ), who originally had the idea of doing a 'two-sided' version of the Jones subfactors. The braid representations can be obtained by using the representation theory of $q$-deformations of the enveloping algebras of the classical Lie algebras, a fact that is essential for computing the weight vectors for the trace $([\mathrm{W}-1],[\mathrm{W}-2],[\mathrm{W}-3])$. In these cases, the weight vectors are given in terms of the $q$-dimensions, and the index for the pair, in the case $m=0$, is the sum of the square of the $q$-dimensions. The explicit formulas are computed in section 4 .

It turns out that for $m=0$, the asymptotic pairs of subfactors (in the sense of Ocneanu, see $[\mathrm{O}]$ ) corresponding to the one-sided constructions of subfactors associated to the Lie types A,B,C,D (i.e., the Wenzl subfactors and Jones' in the $s l(2)$ case) coincide with our new examples, their two-sided versions. We prove this fact for the B,C,D types at the end of section 4.3, and leave out the type A case for later.

The paper is organized as follows:

In section 2 we give some basic definitions and mention some results to be used in the later sections. The general construction of pairs of subfactors is done in section 3: first the assumptions needed on the braid representations, then the definition of the pairs and finally a general formula for the index and the relative commutant. In section 4 we apply the general construction to the natural examples of unitary braid representations associated with Lie theory, mentioned above, and we work out explicit formulas for the index of these pairs.

Acknowledgements. This is a version of my thesis. I would like to thank my advisor, Fred Goodman, for his invaluable help and support. I would also like to thank Hans Wenzl for his very useful suggestions and many math discussions. I am also very grateful to Ryszard Nest and Uffe Haagerup for making it possible for me to visit the Mathematics Institutes at Copenhagen and Odense, as well as to the hospitality of The Fields Institute. Finally, I would like to thank the University of Iowa for having awarded me the T. A. Cleary International Dissertation Research Award.

\section{Preliminaries}

Let $A$ and $B$ be finite dimensional $\mathrm{C}^{*}$-algebras. We can write them as finite direct sums of matrix algebras, $A=\bigoplus_{i=1, \ldots, n} A_{i}$ and $B=\bigoplus_{j=1, \ldots, m} B_{j}$, with $A_{i} \cong M_{n_{i}}(\mathbb{C})$ and $B_{j} \cong M_{m_{j}}(\mathbb{C})$. If $A$ is included in $B$ with the same identity, we define the inclusion matrix as follows. A simple $B_{j}$ module can be regarded as an $A$ module. Denote by $g_{i j}$ the number of simple $A_{i}$ modules in its decomposition into simple $A$ modules. The matrix $G=\left(g_{i j}\right)$ is called the inclusion matrix for the pair $A \subseteq B$. One can encode the inclusion matrix by the Bratteli diagram: place two sets of points in parallel lines, one labeled by $I=\{1, \ldots, n\}$ and the other by $J=\{1, \ldots, m\}$. The points of each set correspond to minimal central idempotents. We join the $i$-th point for $A$ and the $j$-th point for $B$ by $g_{i j}$ edges. 
We shall need the concept of periodicity for an ascending sequence of inclusions $\ldots \subseteq C_{n} \subseteq C_{n+1} \subseteq \ldots$ of finite dimensional $\mathrm{C}^{*}$-algebras . A sequence $\left(C_{n}\right)$ as above is called periodic with period $k$, where $k$ is a positive integer, if there exists a positive integer $n_{0}$ such that for $n \geq n_{0}$ the inclusion matrix for the pair $C_{n} \subseteq C_{n+1}$ coincides with that for $C_{n+k} \subseteq C_{n+1+k}$ (after a possible relabeling of the minimal central projections). We shall include in this definition the additional requirement that the inclusion matrix $H_{n}$ for the pair $C_{n} \subseteq C_{n+k}$ should also be primitive (i.e., there exists some $l \in \mathbb{N}$ such that $H_{n}^{l}$ has only strictly positive entries). If $\left(C_{n}\right)$ is periodic it follows by Perron-Frobenius theory that there exists a unique positive trace on $\bigcup C_{n}$, which is moreover faithful; positivity and faithfulness mean that the trace of each projection is strictly positive.

Now, let $\left(C_{n}\right)$ and $\left(D_{n}\right)$ be two periodic sequences, both with period $k$, such that $C_{n} \subseteq D_{n}$ for all $n \in \mathbb{N}$. The pair of sequences $\left(C_{n}\right) \subseteq\left(D_{n}\right)$ is said to be periodic with period $k$ if in addition the inclusion matrices for $C_{n} \subseteq D_{n}$ and for $C_{n+k} \subseteq D_{n+k}$ coincide for large $n$.

Let $\left(C_{n}\right) \subseteq\left(D_{n}\right)$ be a pair of ascending sequences, and consider a faithful positive trace $t r$ on the inductive limit $\bigcup D_{n}$ with faithful restriction to $\bigcup C_{n}$. The pair $\left(C_{n}\right) \subseteq\left(D_{n}\right)$ is said to satisfy the commuting square condition if for all large $n$ the following diagrams commute:

$$
\begin{gathered}
D_{n} \hookrightarrow D_{n+1} \\
E_{C_{n}} \downarrow \downarrow E_{C_{n+1}} \\
C_{n} \hookrightarrow C_{n+1}
\end{gathered}
$$

i.e., if $E_{C_{n+1}}\left(D_{n}\right) \subseteq C_{n}$, where $E_{C_{n}}$ and $E_{C_{n+1}}$ are the unique trace preserving conditional expectations onto $C_{n}$ and $C_{n+1}$ respectively. (The conditional expectation onto $D_{n}$ is determined by the condition $\operatorname{tr}\left(E_{D_{n}}(x) y\right)=\operatorname{tr}(x y)$ for all $y \in D_{n}$; see [Po1], or [GHJ, Ch.IV].)

Here we state some no doubt well known combinatorial results that will be needed in the last section:

Lemma 2.1. ( $i)$ If $A \in M_{k}(\mathbb{C})$ is given by coefficients $a_{i j}=\frac{1}{i+j}\left(\begin{array}{c}2(i+j-1) \\ i+j-1\end{array}\right)$, then $\operatorname{det} A=1$

(ii) If $A \in M_{k}(\mathbb{C})$ is given by coefficients $a_{i j}=\left(\begin{array}{c}2(i+j-1) \\ i+j-1\end{array}\right)$, then $\operatorname{det} A=2^{k}$.

(iii) If $A \in M_{k}(\mathbb{C})$ is given by coefficients $a_{i j}=\left(\begin{array}{c}2(i+j-2) \\ i+j-2\end{array}\right)$, then $\operatorname{det} A=2^{k-1}$.

Proof. (i) It is enough to show that $A=L L^{t}$, where $L \in M_{k}(\mathbb{C})$ is a lower triangular matrix with ones in the diagonal. Let $L \in M_{k}(\mathbb{C})$ be given by

$$
L_{i j}:= \begin{cases}\left\{\begin{array}{c}
2 i-1 \\
i-j
\end{array}\right\} & \text { for } i \geq j, \\
0 & \text { otherwise, }\end{cases}
$$

where

$$
\left\{\begin{array}{l}
n \\
s
\end{array}\right\}:= \begin{cases}\left(\begin{array}{l}
n \\
s
\end{array}\right)-\left(\begin{array}{c}
n \\
s-1
\end{array}\right) & \text { if } s \geq 1, \\
1 & \text { for } s=0 .\end{cases}
$$

Recall that $c(n):=\frac{1}{n+1}\left(\begin{array}{c}2 n \\ n\end{array}\right)$ is equal to the number of monotonically increasing (MI) paths in $\mathbb{N}_{0} \times \mathbb{N}_{0}$ from $(0,0)$ to $(n, n)$ that stay on or above the diagonal; in particular, $a_{i j}=c(i+j-1)$. One can also show that for $s \leq i, L_{i s}$ is the number of MI paths from $(0,0)$ to $(i-s, i+s-1)$ that stay on or above the diagonal. 
For showing that in fact $A=L L^{t}$, i.e., $c(i+j-1)=\sum_{s=1}^{k} L_{i s} L_{j s}$, one notes that any MI path from $(0,0)$ to $(i+j-1, i+j-1)$ that stays on or above the diagonal passes through a node $(i-s, i+s-1)$ for some $s \in\{1, \ldots, i\}$, and that for $i \leq j$, $1 \leq s \leq i, L_{j s}$ also coincides with the number of MI paths from $(i-s, i+s-1)$ to $(i+j-1, i+j-1)$ that stay on or above the diagonal.

The proofs for $(i i)$ and $(i i i)$ use the same arguments as the one for $(i)$.

\section{A CONSTRUCtion OF SUBFACTORS}

3.1 Assumptions. To define the pairs of hyperfinite $\mathrm{II}_{1}$ factors we start with certain unitary representations of the braid group as in Wenzl's work [W-1], [W-2], [W-3]. Recall that the braid group $\mathbf{B}_{n}$ on $n$ strands is defined by generators $\sigma_{1}, \ldots, \sigma_{n-1}$ and the braid relations

$\left(B_{1}\right) \sigma_{i+1} \sigma_{i} \sigma_{i+1}=\sigma_{i} \sigma_{i+1} \sigma_{i}$, for $i=1, \ldots, n-2$,

$\left(B_{2}\right) \sigma_{i} \sigma_{j}=\sigma_{j} \sigma_{i}$, for $|i-j| \geq 2$.

A geometric picture of the standard generator $\sigma_{i}$ is given by the following diagram:

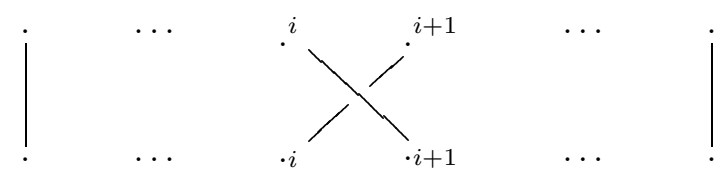

and multiplication is given by concatenation of such diagrams (see [Bi] for more details). $\mathbf{B}_{n}$ is embedded into $\mathbf{B}_{n+1}$ by adding one vertical strand at the end of each generator of $\mathbf{B}_{n}$. Denote $\bigcup \mathbf{B}_{n}$ by $\mathbf{B}_{\infty}$.

We shall work with representations $\rho$ of $\mathbb{C B}_{\infty}$ that satisfy the following properties:

(i) $\rho$ is locally finite dimensional: By this we mean that for every $n \in \mathbb{N}, \rho\left(\mathbb{C B}_{n}\right)$ is a finite dimensional $\mathrm{C}^{*}$-algebra, so that we can write $\rho\left(\mathbb{C} \mathbf{B}_{n}\right) \cong \bigoplus_{\lambda \in \Lambda_{n}} M_{a_{\lambda}}(\mathbb{C})$, for some index set $\Lambda_{n}$. Set $A_{n}=\rho\left(\mathbb{C B}_{n}\right)$.

(ii) $\rho$ is unitary: $g_{i}:=\rho\left(\sigma_{i}\right)$ is unitary for all $i$.

(iii) The ascending sequence of finite dimensional $\mathrm{C}^{*}$-algebras $\left(A_{n}\right)=\left(\rho\left(\mathbb{C B}_{n}\right)\right)$ is periodic, in the sense defined in the preliminaries.

(iv) Any element $x \in A_{n+1}$ can be written as a sum of elements $a g_{n}^{ \pm 1} b+c$ with $a, b, c \in A_{n}$.

(v) The unique positive faithful trace $\operatorname{tr}$ on $\bigcup A_{n}$ has the Markov property:

$$
\operatorname{tr}\left(g_{n}^{ \pm 1} x\right)=\eta \operatorname{tr}(x) \text { for all } x \in A_{n} \text {, for all } n,
$$

where $\eta$ is a fixed complex number. Given condition (iv), the Markov condition implies the multiplicativity property for the trace:

$$
\operatorname{tr}(x y)=\operatorname{tr}(x) \operatorname{tr}(y)
$$

if $x$ and $y$ are in subalgebras generated by disjoint subsets of generators $g_{i}^{ \pm 1}$.

(vi) Existence of a projection $p$ with the contraction property: $p \in A_{k}$ has the contraction property if for all $n \in \mathbb{N}$,

$$
p A_{n+k} p \cong p A_{k+1, n+k} \cong A_{k+1, n+k},
$$

where $A_{s, t}$ is the algebra generated by $\left\{g_{s}, \ldots, g_{t-1}\right\}$. Note that since we already have the multiplicative property of the trace by (iv) and (v), the second isomorphism above is always true. 
Given a locally finite representation $\rho$ of the braid group, one has an associative, commutative, graded product on $\bigoplus_{n} K_{0}\left(A_{n}\right)$ defined as follows. See [GW] for details. For projections $x \in A_{n}$ and $y \in A_{m}$ define $x \otimes y=x \operatorname{shift}_{n}(y) \in A_{n+m}$, where shift ${ }_{n}: \mathbb{C B}_{m} \rightarrow \mathbb{C B}_{n+m}$ is determined by $\sigma_{i} \mapsto \sigma_{i+n}$. Then $[x] \otimes[y]=[x \otimes y]$ defines the multiplication in $\bigoplus_{n} K_{0}\left(A_{n}\right)$. Denote the structure constants of this multiplication by $c_{\lambda \mu}^{\nu}$. That is, if $p_{\lambda}$ and $p_{\mu}$ are minimal projections in the classes labelled by $\lambda \in \Lambda_{n}$ and $\mu \in \Lambda_{m}$, then

$$
\left[p_{\lambda}\right] \otimes\left[p_{\mu}\right]=\sum_{\nu \in \Lambda_{n+m}} c_{\lambda \mu}^{\nu}\left[p_{\nu}\right]
$$

We have the following conditions equivalent to the existence of a projection with the contraction property; two of them are found in [W-3]:

Lemma 3.1.1. With the same notation as throughout this section, the following are equivalent:

(a) There exists a projection $p \in A_{k}$ with the contraction property, i.e., for all $n \in \mathbb{N}, p A_{n+k} p \cong p A_{k+1, n+k} \cong A_{k+1, n+k}$, where $A_{s, t}$ is the algebra generated by $\left\{g_{s}, \ldots, g_{t-1}\right\}$.

(b) There exists a projection $p \in A_{k}$ such that for every minimal projection $p_{\lambda} \in A_{n}$, and for all $n \in \mathbb{N}$, the projection $p \otimes p_{\lambda}$ remains minimal in $A_{n+k}$. Moreover, if $\lambda \neq \lambda^{\prime}$ then $p \otimes p_{\lambda}$ and $p \otimes p_{\lambda^{\prime}}$ are not equivalent.

(c) For all $n \in \mathbb{N}_{0}$ there exists an injective map $j: \Lambda_{n} \rightarrow \Lambda_{n+k}$ that preserves the structure coefficients for the multiplication in $\bigoplus_{n} K_{0}\left(A_{n}\right)$, that is, such that $c_{\lambda \mu}^{\nu}=c_{j(\lambda) \mu}^{j(\nu)}=c_{\lambda j(\mu)}^{j(\nu)}$ for all $\lambda \in \Lambda_{n}, \mu \in \Lambda_{m}, \nu \in \Lambda_{n+m}$, and also such that $c_{\lambda j(\mu)}^{\epsilon}=0$ if $\epsilon \notin j\left(\Lambda_{n+m}\right)$. (Here, $\Lambda_{0}:=\{[\varnothing]\}$, 'the empty diagram', and $p_{[\varnothing]}:=i d$, so that $c_{[\varnothing] \lambda}^{\mu}=\delta_{\lambda, \mu}$, for $\lambda, \mu \in \Lambda_{n}$.)

Proof. $(a) \Rightarrow(b)$ : By $(a)$, the following maps are isomorphisms: the inclusion $\iota$ : $p A_{k+1, n+k} \rightarrow p A_{n+k} p$, since it is injective, and the map $\phi: A_{k+1, n+k} \rightarrow p A_{k+1, n+k}$ given by $a \mapsto p a$, since it is surjective. Also, the shift $\operatorname{shift}_{k}: A_{n} \rightarrow A_{k+1, n+k}$ is an isomorphism. Therefore, the composition map $\psi=\iota \circ \phi \circ \operatorname{shift}_{k}: A_{n} \rightarrow p A_{n+k} p$ is an isomorphism. Hence, for any minimal idempotent $p_{\lambda} \in A_{n}$, we shall have that $\psi\left(p_{\lambda}\right)=p \otimes p_{\lambda}$ is minimal in $p A_{n+k} p$. But then $q:=p \otimes p_{\lambda}$ remains minimal in $A_{n+k}$, since if $q=a+b$, where $a, b \in A_{n+k}$ are projections with $a b=0$, then it follows that $a, b \in p A_{n+k} p$, and so $a=0$ or $b=0$.

If $\lambda \neq \lambda^{\prime}$ then $p_{\lambda}$ and $p_{\lambda^{\prime}}$ are not equivalent, and so $p \otimes p_{\lambda}$ and $p \otimes p_{\lambda^{\prime}}$ are not equivalent in $p A_{n+k} p$. But then they remain non-equivalent in $A_{n+k}$ : if $v$ were a partial isometry that implements the equivalence in $A_{n+k}, p v p$ would implement an equivalence in $p A_{n+k} p$.

(b) $\Rightarrow(c)$ : For $n \in \mathbb{N}$ define the map $j: \Lambda_{n} \rightarrow \Lambda_{n+k}$ in the following way. For $\lambda \in \Lambda_{n}$, there exists a unique $\mu:=j(\lambda) \in \Lambda_{n+k}$ such that $[p] \otimes\left[p_{\lambda}\right]=\left[p_{\mu}\right]$, according to $(b)$. Since $\left[p \otimes p_{\lambda}\right] \neq\left[p \otimes p_{\lambda^{\prime}}\right]$ for $\lambda \neq \lambda^{\prime}$, then $j$ is injective. Also, (b) implies that $p \in A_{k}$ is minimal, and so $p=p_{\lambda_{0}}$ for some $\lambda_{0} \in \Lambda_{k}$. Define $j: \Lambda_{0} \rightarrow \Lambda_{k}$ by $j([\varnothing])=\lambda_{0}$. Now use the associativity of the multiplication in $\bigoplus K_{0}\left(A_{n}\right)$ as follows:

$$
\left[p_{\lambda_{0}}\right] \otimes\left(\left[p_{\lambda}\right] \otimes\left[p_{\mu}\right]\right)=[p] \otimes \sum_{\nu \in \Lambda_{n+m}} c_{\lambda \mu}^{\nu}\left[p_{\nu}\right]=\sum_{\nu \in \Lambda_{n+m}} c_{\lambda \mu}^{\nu}\left[p_{j(\nu)}\right],
$$


and

$$
\left(\left[p_{\lambda_{0}}\right] \otimes\left[p_{\lambda}\right]\right) \otimes\left[p_{\mu}\right]=\left[p_{j(\lambda)}\right] \otimes\left[p_{\mu}\right]=\sum_{\epsilon \in \Lambda_{n+m+k}} c_{j(\lambda) \mu}^{\epsilon}\left[p_{\epsilon}\right] .
$$

Comparing these two expressions gives

$$
c_{\lambda \mu}^{\nu}=c_{j(\lambda) \mu}^{j(\nu)} \text { and } c_{j(\lambda) \mu}^{\epsilon}=0 \text { if } \epsilon \notin j\left(\Lambda_{n+m}\right) .
$$

$(c) \Rightarrow(a)$ : Let $p \in A_{k}$ be a projection in the class labelled by $j([\varnothing])=\lambda_{0}$. It follows from $(c)$ that $[p] \otimes\left[p_{\lambda}\right]=\left[p_{j(\lambda)}\right]$ for $n \in \mathbb{N}$ and $\lambda \in \Lambda_{n}$. Thus, the class of $p$ in $A_{n+k}$ is

$$
\left[p \otimes 1_{n}\right]=\sum_{\lambda \in \Lambda_{n}} \operatorname{dim}(\lambda)[p] \otimes\left[p_{\lambda}\right]=\sum_{\lambda \in \Lambda_{n}} \operatorname{dim}(\lambda)\left[p_{j(\lambda)}\right],
$$

where the minimal ideal in $A_{n}$ labelled by $\lambda \in \Lambda_{n}$ is isomorphic to $M_{\operatorname{dim}(\lambda)}(\mathbb{C})$. It follows immediately that $p A_{n+k} p \cong A_{n}$. Since shift $k: A_{n} \rightarrow A_{k+1, n+k}$ is an isomorphism, we also have that $p A_{n+k} p \cong p A_{k+1, n+k}$.

Remarks. Assume that $\left(A_{n}\right)$ has periodicity $k$ and that there exists a projection $p \in A_{k^{\prime}}$ with the contraction property.

(1) The periodicity condition on the ascending sequence $\left(A_{n}\right)$ forces the injective map $j: \Lambda_{n} \rightarrow \Lambda_{n+k^{\prime}}$ (from the contraction property) to be a bijection for large $n \in \mathbb{N}$.

(2) The sequence $\left(A_{n}\right)$ is also $k^{\prime}$-periodic, so one can assume without loss of generality that $k=k^{\prime}$.

(3) Let $I$ be given by the set $\left\{k \in \mathbb{N}\right.$ such that $\left(A_{n}\right)$ is $k$-periodic $\}$. Let $k_{0}$ be given by $\min I$. Then $I=\left\{n k_{0}\right.$ such that $\left.n \in \mathbb{N}\right\}$.

(4) $p^{\otimes s} \in A_{s k^{\prime}}$ has the contraction property for all $s \in \mathbb{N}$.

Proof of the remarks. (1) The $k$-periodicity of $\left(A_{n}\right)$ implies that $\left|\Lambda_{n}\right|=\left|\Lambda_{n+k}\right|$ for large $n$. By the contraction property there exist $k^{\prime} \in \mathbb{N}$ and an injective map $j: \Lambda_{n} \rightarrow \Lambda_{n+k^{\prime}}$ for $n \in \mathbb{N}_{0}$. Then we shall have that the injective map $j^{(k)}:=$ $j \circ j \circ \cdots \circ j: \Lambda_{n} \rightarrow \Lambda_{n+k k^{\prime}}$ is a bijection for large $n$, since $\left|\Lambda_{n}\right|=\left|\Lambda_{n+k k^{\prime}}\right|$. Hence, the map $j: \Lambda_{n} \rightarrow \Lambda_{n+k^{\prime}}$ will also be surjective for large $n$ since we have the inequalities $\cdots \leq\left|\Lambda_{n}\right| \leq\left|\Lambda_{n+k^{\prime}}\right| \leq \cdots \leq\left|\Lambda_{n+k k^{\prime}}\right| \leq \cdots$.

(2) By the previous remark, the map $j: \Lambda_{n} \rightarrow \Lambda_{n+k^{\prime}}$ is bijective for large $n$. But this will also imply $k^{\prime}$-periodicity: By $(c)$ of Lemma 3.1.1, the inclusion matrix for the pair $A_{n} \subseteq A_{n+1}, \quad\left(g_{\lambda \mu}\right)_{\lambda \in \Lambda_{n}, \mu \in \Lambda_{n+1}}=\left(c_{\lambda[1]}^{\mu}\right)_{\lambda \in \Lambda_{n}, \mu \in \Lambda_{n+1}}$, is preserved by $j$, i.e., $g_{\lambda \mu}=g_{j(\lambda) j(\mu)}$ for all $\lambda \in \Lambda_{n}, \mu \in \Lambda_{n+1}$, where the unique element of $\Lambda_{1}$ is denoted by [1] (the "Young" diagram consisting of one box: we shall see later that in our examples the label sets will consist of Young diagrams). Since $j\left(\Lambda_{n}\right)=\Lambda_{n+k^{\prime}}$ for large $n$, then the inclusion matrix for $A_{n+k^{\prime}} \subseteq A_{n+k^{\prime}+1}$ is given by $\left(g_{\epsilon \nu}\right)_{\epsilon \in \Lambda_{n+k^{\prime}}, \nu \in \Lambda_{n+k^{\prime}+1}}=\left(g_{j(\lambda) j(\mu)}\right)_{\lambda \in \Lambda_{n}, \mu \in \Lambda_{n+1}}$, and so $\left(A_{n}\right)$ is $k^{\prime}$-periodic.

(3) It is enough to show that if $s, t \in I$ and $s>t$ then $s-t \in I$; and this follows easily from the definition of periodicity.

(4) Follows easily by induction on $s \in \mathbb{N}$.

3.2 The construction. We proceed with the construction of our pairs of factors. We can extend the representations $\rho$ of $\mathbb{C B}_{\infty}$ satisfying the conditions (i)-(vi) in section 3.1 to the two-sided infinite braid group. By that, we mean the inductive limit of the two-sided $n$-braid groups given by generators $\sigma_{-n+1}, \sigma_{-n+2}, \ldots, \sigma_{n-1}$ 
and the usual braid relations. Then, fix a non-negative integer $m$ and define, for $n \geq m+2$,

$$
\begin{aligned}
& D_{n}:=\rho\left(\left\langle\sigma_{-n+1}, \sigma_{-n+2}, \ldots, \sigma_{n-2}, \sigma_{n-1}\right\rangle\right)=\left\langle g_{-n+1}, g_{-n+2}, \ldots, g_{n-2}, g_{n-1}\right\rangle, \\
& C_{n}:=\rho\left(\left\langle\sigma_{-n+1}, \ldots, \sigma_{-1}, \sigma_{m+1}, \ldots, \sigma_{n-1}\right\rangle\right)=\left\langle g_{-n+1}, \ldots, g_{-1}, g_{m+1}, \ldots, g_{n-1}\right\rangle .
\end{aligned}
$$

By the map $g_{i} \mapsto g_{n+i}$ (relabelling of the indices of the generators) and by the trace preserving automorphism $g_{-i} g_{j} \mapsto g_{n-i} \otimes g_{j}$ we have

$$
\begin{gathered}
D_{n} \cong A_{2 n}=\rho\left(\mathbb{C B}_{2 n}\right), \\
C_{n} \cong A_{n} \otimes A_{n-m} .
\end{gathered}
$$

Lemma 3.2.1. The pair of ascending sequences $\left(C_{n}\right) \subseteq\left(D_{n}\right)$ is periodic.

Proof. First we shall show that the periodicity for each of the two sequences $\left(C_{n}\right)$ and $\left(D_{n}\right)$ is an easy consequence of our periodicity hypothesis for $\left(A_{n}\right)$. The inclusion matrix for $C_{n} \subseteq C_{n+1}$ is the same as that for $A_{n} \otimes A_{n-m} \subseteq A_{n+1} \otimes A_{n-m+1}$, by (3.2.2). Thus, $\left(C_{n}\right)$ is $k$-periodic, where $k$ is the period of $\left(A_{n}\right)$. The inclusion matrix for $C_{n} \subseteq C_{n+k}$ is primitive because so is the one for $A_{n} \subseteq A_{n+k}$ (the coefficients of $(G \otimes G)^{s}$ are positive when those of $G^{s}$ are positive).

By (3.2.1) the inclusion matrix for the pair $D_{n} \subseteq D_{n+1}$ is conjugate to

$$
\left\langle g_{2}, \ldots, g_{2 n}\right\rangle \subseteq\left\langle g_{1}, \ldots, g_{2 n+1}\right\rangle .
$$

Inserting above an intermediate algebra, $\left\langle g_{2}, \ldots, g_{2 n+1}\right\rangle$, we have the inclusions

$$
\left\langle g_{2}, \ldots, g_{2 n}\right\rangle \subseteq\left\langle g_{2}, \ldots, g_{2 n+1}\right\rangle \subseteq\left\langle g_{1}, \ldots, g_{2 n+1}\right\rangle .
$$

The inclusion at the first stage in (3.2.3) is conjugate to $A_{2 n} \subseteq A_{2 n+1}$ and that at the second stage is conjugate, by an inner automorphism of $A_{2 n+2}$ (the 'half-twist' which sends $g_{i}$ to $\left.g_{2 n+2-i}\right)$, to $A_{2 n+1} \subseteq A_{2 n+2}$. Hence, we obtain that $D_{n} \subseteq D_{n+1}$ is conjugate to $A_{2 n} \subseteq A_{2 n+2}$, so that $\left(D_{n}\right)$ is $k$-periodic, and the primitivity condition for the inclusion matrices follows from the same condition for the sequence $\left(A_{n}\right)$.

It remains to show that for large $n$ the inclusion for $C_{n} \subseteq D_{n}$ coincides with that for $C_{n+k} \subseteq D_{n+k}$. For this we shall use the projection with the contraction property. By remark (2) in section 3.1 we assume that $k$ is the same for the periodicity and the contraction properties. The inclusion for $C_{n} \subseteq D_{n}$ is conjugate to

$$
A_{n} \otimes A_{n-m} \subseteq A_{2 n} .
$$

We split this inclusion into two steps:

$$
A_{n} \otimes A_{n-m} \subseteq A_{n} \otimes A_{n} \subseteq A_{2 n} .
$$

The inclusion at the first stage in (3.2.4) coincides with that for $A_{n+k} \otimes A_{n+k-m} \subseteq$ $A_{n+k} \otimes A_{n+k}$ by periodicity of $\left(A_{n}\right)$. The inclusion data for the pair $A_{n} \otimes A_{n} \subseteq A_{2 n}$ are precisely the structure constants for the multiplication $K_{0}\left(A_{n}\right) \times K_{0}\left(A_{n}\right) \rightarrow$ $K_{0}\left(A_{2 n}\right)$, that is, $g_{(\lambda \mu) \nu}=c_{\lambda \mu}^{\nu}$. But these coincide with the structure constants for the multiplication $K_{0}\left(A_{n+k}\right) \times K_{0}\left(A_{n+k}\right) \rightarrow K_{0}\left(A_{2 n+2 k}\right)$,

$$
c_{j(\lambda) j(\mu)}^{j^{2}(\nu)}=c_{\lambda \mu}^{\nu},
$$

by two applications of the equation $c_{\lambda j(\mu)}^{j(\nu)}=c_{\lambda \mu}^{\nu}$, from Lemma 3.1.1, $(c)$, and remark (1) in section 3.1. Thus, the inclusion matrix at the second stage in (3.2.4), 
$A_{n} \otimes A_{n} \subseteq A_{2 n}$, also coincides with that for $A_{n+k} \otimes A_{n+k} \subseteq A_{2 n+2 k}$. It follows that the inclusion $C_{n} \subseteq D_{n}$ is the same as that for $C_{n+k} \subseteq D_{n+k}$ for large $n$, and finally that $\left(C_{n}\right) \subseteq\left(D_{n}\right)$ is a $k$-periodic pair.

It follows from periodicity that the trace on $D_{\infty}=\bigcup_{n} D_{n}$ and also on $C_{\infty}=$ $\bigcup_{n} C_{n}$ is faithful and unique. We define $D$ and $C$ to be the weak closure of $D_{\infty}$ and $C_{\infty}$ in the GNS representation with respect to the trace. Thus, we obtain a pair of hyperfinite $\mathrm{II}_{1}$ factors $C \subseteq D$.

Lemma 3.2.2. The pair of ascending sequences $\left(C_{n}\right) \subseteq\left(D_{n}\right)$ has the commuting square property.

Proof. This will be a consequence of having conditions (iv) and (v). It can be shown easily by inserting intermediate algebras $\tilde{C}_{n}:=\left\langle C_{n}, g_{n}\right\rangle$ and $\tilde{D}_{n}:=\left\langle D_{n}, g_{n}\right\rangle$.

\subsection{A formula for the index.}

Proposition 3.3.1. The index for the pair of subfactors $C \subseteq D$ defined in section 3.2 is given by the expression

$$
[D: C]=\left\|\vec{d}^{(n)}\right\|^{2} W^{m},
$$

for large $n$, where $W^{m}$ is the index for the 'one-sided' pair, that is, for the pair $\left\langle g_{m+1}, \ldots, g_{n}, \ldots\right\rangle^{\prime \prime} \subseteq\left\langle g_{1}, \ldots, g_{n}, \ldots\right\rangle^{\prime \prime}$, and $\vec{d}^{(n)}$ is a multiple of the weight vector $\vec{t}^{(n)}$ for the trace on $A_{n}=\rho\left(\mathbb{C B}_{n}\right)$, normalized as follows: $\vec{d}_{j^{s}([\varnothing])}^{(k s)}=1$ for large $s$ and $\vec{d}^{(n)}=W^{-1 / 2} G_{n} \vec{d}^{(n+1)}$, where $G_{n}$ denotes the inclusion matrix for $A_{n} \subseteq A_{n+1}$.

Proof. By [W-1, Theorem 1.5, (iii)], the index for the pair $C \subseteq D$ is

$$
[D: C]=\frac{\left\|\vec{s}^{(n)}\right\|^{2}}{\left\|\vec{v}^{(n)}\right\|^{2}}
$$

for large $n$, where $\vec{s}^{(n)}$ and $\vec{v}^{(n)}$ are the weight vectors for the trace restricted to $C_{n}$ and $D_{n}$ respectively. Because of the trace preserving identifications (3.2.1) and (3.2.2), and because of the multiplicativity of the trace, we have that $s_{i, j}^{(n)}=$ $t_{i}^{(n)} t_{j}^{(n-m)}$, and $\vec{v}^{(n)}=\vec{t}^{(2 n)}$, where $\vec{t}^{(n)}$ is the weight vector for the trace on $A_{n}$. Hence, we have

$$
\begin{aligned}
{[D: C] } & =\frac{\left\|\vec{s}^{(n)}\right\|^{2}}{\left\|\vec{v}^{(n)}\right\|^{2}}=\frac{\left\|\vec{t}^{(n)}\right\|^{2}\left\|\vec{t}^{(n-m)}\right\|^{2}}{\left\|\vec{t}^{(2 n)}\right\|^{2}} \\
& =\left\|\vec{t}^{(n)}\right\|^{2} \prod_{i=0}^{n+m-1} \frac{\left\|\vec{t}^{(n-m+i)}\right\|^{2}}{\left\|\vec{t}^{(n-m+i+1)}\right\|^{2}}
\end{aligned}
$$

But the index for the one-sided pair $N:=\left\langle g_{2}, \ldots, g_{n}, \ldots\right\rangle^{\prime \prime} \subseteq M:=\left\langle g_{1}, \ldots, g_{n}, \ldots\right\rangle^{\prime \prime}$ is given by

$$
W:=\frac{\left\|\vec{t}^{(r)}\right\|^{2}}{\|\vec{t}(r+1)\|^{2}}
$$

for large $r$, by Wenzl's formula. Then, for large $n$,

$$
[D: C]=\left\|\vec{t}^{(n)}\right\|^{2} W^{n+m}=\left(W^{n}\left\|\vec{t}^{(n)}\right\|^{2}\right) W^{m} .
$$


Observe that by (3.3.1), $W^{m}$ is the index for $\left\langle g_{m+1}, \ldots, g_{n}, \ldots\right\rangle^{\prime \prime} \subseteq\left\langle g_{1}, \ldots, g_{n}, \ldots\right\rangle^{\prime \prime}$. If we define the vectors

$$
\vec{d}^{(n)}:=\left(W^{1 / 2}\right)^{n} \vec{t}^{(n)},
$$

we can write

$$
[D: C]=\left\|\vec{d}^{(n)}\right\|^{2} W^{m},
$$

independent of $n$, for large $n$. For large $n$ the vectors $\vec{d}^{(n)}$ are in fact the PerronFrobenius eigenvectors for the matrices $H_{n}:=G_{n} G_{n+1} \ldots G_{n+k-1}$, with eigenvalue equal to $W^{k / 2}$, by Perron-Frobenius theory and Wenzl's index formula. See [GHJ] and [W-1]. It follows from (3.3.3) and from the fact that $\vec{t}^{(n)}=G_{n} \vec{t}^{(n+1)}$ that $\vec{d}^{(n)}=W^{-1 / 2} G_{n} \vec{d}^{(n+1)}$ for large $n$.

By (3.3.4), it remains to check that $\vec{d}_{j^{s}([\varnothing])}^{(n)}=1$ if $n$ is a large multiple of $k$, i.e., $n=k s$ for large $s$. If $p \in A_{k}$ has the contraction property, by remark (4) the projection $p^{\otimes s}$ has the contraction property as well. Furthermore, one can show that $\operatorname{tr}(p)=W^{-k / 2}$, as follows:

We have a bijection $j: \Lambda_{n} \rightarrow \Lambda_{n+k}$ of labels for minimal central idempotents such that the map $\left[p_{\lambda}\right] \mapsto[p] \otimes\left[p_{\lambda}\right]=\left[p_{j(\lambda)}\right]$ is a bijection between classes of minimal idempotents of $A_{n}$ and $A_{n+k}$. Moreover,

$$
\operatorname{tr}\left(p_{j(\lambda)}\right)=\operatorname{tr}\left(p \otimes p_{\lambda}\right)=\operatorname{tr}(p) \operatorname{tr}\left(p_{\lambda}\right)
$$

and therefore,

$$
W^{k}=\frac{\left\|\vec{t}^{(n)}\right\|^{2}}{\left\|\vec{t}^{(n+k)}\right\|^{2}}=\frac{1}{\operatorname{tr}(p)^{2}}
$$

so it follows that

$$
\begin{aligned}
\vec{d}_{j^{s}([\varnothing])}^{(k s)} & =\left(W^{1 / 2}\right)^{k s} \vec{t}_{j^{s}([\varnothing])}^{(k s)}=\left(W^{1 / 2}\right)^{k s} \operatorname{tr}\left(p_{j^{s}([\varnothing])}\right) \\
& =\left(W^{1 / 2}\right)^{k s} \operatorname{tr}\left(p^{\otimes s}\right)=\left(W^{1 / 2}\right)^{k s} \operatorname{tr}(p)^{s}=1 .
\end{aligned}
$$

The last part of the proof of the last proposition, (3.3.5), gives us an expression for the index $W$ for the one sided pair of factors $\left\langle g_{2}, \ldots, g_{n}, \ldots\right\rangle^{\prime \prime} \subseteq$ $\left\langle g_{1}, \ldots, g_{n}, \ldots\right\rangle^{\prime \prime}$ in terms of the trace of the projection $p \in A_{k}$ with the contraction property:

Corollary 3.3.2. Under the conditions (i)-(vi), the index $W$ for the one sided pair of factors $\left\langle g_{2}, \ldots, g_{n}, \ldots\right\rangle^{\prime \prime} \subseteq\left\langle g_{1}, \ldots, g_{n}, \ldots\right\rangle^{\prime \prime}$ is given by

$$
W=\operatorname{tr}(p)^{-\frac{2}{k}},
$$

where $p \in A_{k}$ is a projection with the contraction property.

3.4 The relative commutant. Because of the defining relations of the braid group we already have the inclusion $A_{m}=\left\langle g_{1}, \ldots, g_{m-1}\right\rangle \subseteq C^{\prime} \cap D$. We shall show that in fact these algebras coincide.

The factors $C$ and $D$ can be also approximated by a different pair of ascending sequences $\left(\tilde{C}_{n}\right) \subseteq\left(\tilde{D}_{n}\right)$ with the same essential properties (periodicity and the commuting square condition), namely,

$$
\begin{aligned}
& \tilde{C}_{n}:=\left\langle g_{-n+1}, \ldots, g_{-1}, g_{m+1}, \ldots, g_{n+m-1}\right\rangle \cong A_{n} \otimes A_{n}, \\
& \tilde{D}_{n}:=\left\langle g_{-n+1}, \ldots, g_{n+m-1}\right\rangle \cong A_{2 n+m} .
\end{aligned}
$$


Let $p \in A_{k}$ be a projection with the contraction property; then $p^{\otimes s} \in A_{k s}$ has the contraction property as well, for all $s \in \mathbb{N}$ (see remark (4) in section 3.1). Choose $s \in \mathbb{N}$ such that $n=s k$ is large enough, and set $\tilde{p}:=p^{\otimes s} \otimes p^{\otimes s} \in A_{n} \otimes A_{n}$. Since $\tilde{p}$ has the contraction property in $A_{2 n}$, one has

$$
\mathbb{C} \cong \tilde{p}\left(A_{n} \otimes A_{n}\right) \tilde{p} \subseteq \tilde{p} A_{2 n+m} \tilde{p} \cong A_{m} .
$$

Hence, also

$$
\tilde{p}\left(\left(A_{n} \otimes A_{n}\right)^{\prime} \cap A_{2 n+m}\right) \cong A_{m} .
$$

Since the pair $\tilde{C}_{n} \subseteq \tilde{D}_{n}$ is conjugate to $A_{n} \otimes A_{n} \subseteq A_{2 n+m}$, there is a projection $\bar{p} \in \tilde{C}_{n}$ such that $\bar{p}\left(\tilde{C}_{n}^{\prime} \cap \tilde{D}_{n}\right) \cong A_{m}$. Applying Wenzl's estimate in [W-1, Theorem $1.6]$,

$$
\operatorname{dim} C^{\prime} \cap D \leq \operatorname{dim} \bar{p}\left(\tilde{C}_{n}^{\prime} \cap \tilde{D}_{n}\right)=\operatorname{dim} A_{m} .
$$

Finally, $C^{\prime} \cap D \subseteq A_{m}$ and we obtain the desired equality. For $m=0$ or $m=1$, $\operatorname{dim} A_{m}=1$. Hence, $C^{\prime} \cap D \cong \mathbb{C} 1$. We have proved the following

Proposition 3.4.1. The relative commutant $C^{\prime} \cap D$ of the pair of $I I_{1}$ factors $C \subseteq$ $D$ is isomorphic to $A_{m}=\left\langle g_{1}, \ldots, g_{m-1}\right\rangle$, if $m>1$. For $m=0,1$, we have that $C^{\prime} \cap D \cong \mathbb{C} 1$.

Note that in all cases the relative commutant $C^{\prime} \cap D$ is canonically identified with the relative commutant for the one sided pair $\left\langle g_{m+1}, g_{m+2}, \ldots,\right\rangle^{\prime \prime} \subseteq\left\langle g_{1}, g_{2}, \ldots\right\rangle^{\prime \prime}$.

One can use Wenzl's [W-1, Theorem 1.5, (iii)] to define irreducible pairs of the hyperfinite $\mathrm{II}_{1}$ factor from the pair $C \subseteq D$. Namely, if $C^{\prime} \cap D$ is not trivial, one can 'cut down' the pair by a non-zero projection $p \in C^{\prime} \cap D$, and

$$
\left[D_{p}: C_{p}\right]=\operatorname{tr}(p)^{2}[D: C]
$$

where $D_{p}:=\{p d p: d \in D\}^{\prime \prime}$ and $C_{p}:=\{p c p: c \in C\}^{\prime \prime}$. As in Proposition 3.3.1, there is also a nice formulation for the index of the 'reduced' pairs:

Corollary 3.4.2. The index for the reduced pair $C_{p} \subseteq D_{p}$ is given by the expression

$$
\left[D_{p}: C_{p}\right]=\left\|\vec{d}^{(n)}\right\|^{2} S
$$

for large $n$, where $S=\operatorname{tr}(p)^{2} W^{m}$ is the index for the 'one-sided' reduced pair $\left\langle g_{m+1}, \ldots, g_{n}, \ldots\right\rangle_{p}{ }^{\prime \prime} \subseteq\left\langle g_{1}, \ldots, g_{n}, \ldots\right\rangle_{p} "$, and $\vec{d}^{(n)}$ is as in Proposition 3.3.1.

\section{EXAmples}

4.1 Introduction to the examples. In the next sections we shall compute explicitly the index values for the natural examples mentioned in the introduction, where the unitary braid representations considered factor through the algebras $H_{\infty}(q)$ (type $\mathrm{A},[\mathrm{W}-1]$ ) and $C_{\infty}(r, q)$ (types $\mathrm{B}, \mathrm{C}, \mathrm{D},[\mathrm{W}-2]$ ). As it is shown in those papers, unitary braid representations can be obtained via the Drinfeld-Jimbo quantum group approach and have the desired properties for special values of the parameters $r$ and $q$, so that we shall be able to apply our construction. What follows in this section consists of facts which are described in [W-2, §5].

The representations of the braid group are obtained from the representation theory of the $q$ deformations $\mathcal{U}_{q} g$ of the enveloping algebras of the classical Lie algebras $s l(k), s o(2 k), s p(2 k)$ or $s o(2 k+1)$. It is shown in [D] that for each finite dimensional $\mathcal{U}_{q} g$-module $V$ one can obtain a solution of the QYBE, $R \in$ 
$\operatorname{End}(V \otimes V)$. Consider the QYBE solution $R$ for $V \otimes V$, where $V$ is the standard $\mathcal{U}_{q} g$-module (see $[\mathrm{Ji}]$ ), and define $\tilde{R}$ acting on $V \otimes V$ by $\tilde{R}=P \circ R$, where $P(x \otimes y)=y \otimes x$ is the 'flip'. Define the matrices $\tilde{R}_{i}$ in $\otimes^{\infty} M_{k}(\mathbb{C})$, for $i \in \mathbb{N}$, by

$$
R_{i}=1 \otimes \cdots \otimes 1 \otimes \tilde{R} \otimes 1 \otimes \ldots,
$$

acting like $\tilde{R}$ in the $i^{t h}$ and $(i+1)^{s t}$ copies of $V$ and like the identity in the remaining copies of $V$. Then, the algebra $A_{f}(q)$ generated by $\left\{\tilde{R}_{1}, \ldots, \tilde{R}_{f-1}\right\}$ is a quotient of the braid group algebra $\mathbb{C B}_{f}$ factoring through the Hecke algebra $H_{f}(q)$ or through the algebra $C_{f}\left(q^{r(k)}, q\right)$ if we consider the $\mathrm{B}, \mathrm{C}$, D cases instead, where $r(k)$ depends on the Lie type (for $q \neq 0,1$ ).

For $q$ not a root of unity, the algebras $A_{f}(q)$ (for fixed $f$ ) are semisimple and mutually isomorphic. Furthermore, $A_{f}(q)$ is the centralizer of the corresponding quantum universal enveloping algebra $\mathcal{U}_{q} g$ acting on $V^{\otimes f}$, whose image is also semisimple, so that the minimal ideals in $A_{f}(q)$ are labelled by the irreducible representations of $\mathcal{U}_{q} g$ appearing on $V^{\otimes f}$. The algebra $A_{f}(q)$ has a certain canonical trace, the structure trace, and exploiting the duality with $\mathcal{U}_{q} g$ one finds that the weights of the trace are given by

$$
\frac{\chi^{(\lambda)}(D)}{\operatorname{Tr}(D)^{f}}
$$

Here, $\chi^{(\lambda)}$ is the character of an irreducible representation $\lambda$ of the corresponding Lie group $G$, and $D$ is the diagonal matrix $D(q)=q^{\rho}$, where $\rho$ is the half sum of the positive roots of $g$.

When $q$ is a root of unity, $A_{f}(q)$ is non-semisimple (for large $f$ ). Nevertheless, the quotient by the radical of the structure trace, $\pi_{t r}\left(A_{f}(q)\right)$, is semisimple, with minimal ideals labelled by certain 'good' representations of $g$. The weights for the trace on $\pi_{t r}\left(A_{f}(q)\right)$ are still given by the formula (4.1.1). Furthermore $\pi_{t r}\left(A_{f}(q)\right)$ has a $C^{*}$-structure making $g_{i}=\pi_{t r}\left(\tilde{R}_{i}\right)$ unitary, and the weights of the trace are positive.

4.2 Index for subfactors of type A: The Hecke algebra $H_{\infty}(q)$. Let us recall that the finite dimensional Hecke algebra $H_{n}(q)$ is the free complex algebra with generators $1, T_{1}, \ldots, T_{n-1}$, and relations, depending on a parameter $q \in \mathbb{C}$,

(B $\left.B_{1}\right) T_{i+1} T_{i} T_{i+1}=T_{i} T_{i+1} T_{i}$, for $i=1, \ldots, n-2$,

(B) $T_{i} T_{j}=T_{j} T_{i}$, for $|i-j| \geq 2$,

(H) $T_{i}^{2}=(q-1) T_{i}+q$, for $i=1, \ldots, n-1$.

It can be shown by induction that these complex algebras have dimension $n$ !, independent of $q$. Set $H_{\infty}(q)=\bigcup H_{n}(q)$. As mentioned in section 4.1, the interesting cases occur when $q$ is a root of unity. We take $q=e^{ \pm 2 \pi i / l}$, with $l \geq 3$. We shall summarize the parametrizations of the quotients $\pi_{t r}\left(H_{n}(q)\right)$ with $q$ as above, associated with $\mathcal{G}=s l(k)$ for $1<k<l$; see [W-1] for details.

For $k \in \mathbb{N}$, and $k<l$, a $(k, l)$ Young diagram $\lambda$ of size $n$ is a $k$-tuple $\lambda=$ $\left(\lambda_{1}, \ldots, \lambda_{k}\right)$ with $\lambda_{1} \geq \lambda_{2} \geq \ldots \geq \lambda_{k} \geq 0, \lambda_{1}-\lambda_{k} \leq l-k$, and $\sum_{i=1}^{k} \lambda_{i}=n$. We denote the set of $(k, l)$ diagrams of size $n$ by $\Lambda_{n}^{(k, l)}$. We can also regard a $(k, l)$ diagram $\lambda=\left(\lambda_{1}, \ldots, \lambda_{k}\right)$ of size $n$ as $k$ ordered rows of boxes with $\lambda_{i}$ boxes in the $i^{\text {th }}$ row.

For each diagram $\lambda \in \Lambda_{n}^{(k, l)}$ Wenzl defined in [W-1] an irreducible representation $\pi_{\lambda}^{(k, l)}$ of $H_{n}(q)$ acting on finite dimensional vector space $V_{\lambda}$ (whose basis is labelled 
by the so-called $(k, l)$ tableaux $t$ of shape $\lambda$; see [W-1]). Different diagrams in $\Lambda_{n}^{(k, l)}$ give inequivalent representations. Regarding $H_{n-1}(q)$ as the subalgebra of $H_{n}(q)$ generated by $1, T_{1}, \ldots, T_{n-2}$, we obtain the restriction rule $\left.\pi_{\lambda}^{(k, l)}\right|_{H_{n-1}} \cong$ $\bigoplus_{\lambda^{\prime}<\lambda} \pi_{\lambda^{\prime}}^{(k, l)}$, where $\lambda^{\prime}<\lambda$ means that $\lambda$ can be obtained by adding one box to $\lambda^{\prime} \in \Lambda_{n-1}^{(k, l)}$. One can then define a representation $\pi_{n}^{(k, l)}$ of $H_{n}(q)$ by $\pi_{n}^{(k, l)}(x)=$ $\bigoplus_{\lambda \in \Lambda_{n}^{(k, l)}} \pi_{\lambda}^{(k, l)}(x)$, for $x \in H_{n}(q)$. Its restriction to $H_{n-1}(q)$ is equivalent to $\pi_{n-1}^{(k, l)}$. Finally, there is a well defined representation $\pi^{(k, l)}$ of $H_{\infty}(q)$ given by

$$
\pi^{(k, l)}(x)=\pi_{n}^{(k, l)}(x), \quad \text { if } x \in H_{n}(q) .
$$

This representation agrees with $\pi_{t r}$ described in section 4.1. It is locally finite dimensional, unitarizable, and $t r$ is a positive finite Markov trace. The ascending sequence $\left(\pi^{(k, l)}\left(\mathbb{C B}_{n}\right)\right)$ is periodic with period $k$. From [W-1] and [GW] it can be checked that all the conditions (i)-(v) are satisfied by the representations $\pi^{(k, l)}$, and so we can apply our construction. The weight vector for the trace, see [W-1], is given by $\vec{t}^{(n)}=\left(w_{\lambda}\right)_{\lambda \in \Lambda_{n}^{(k, l)}}$, with

$$
\begin{aligned}
w_{\lambda} & =\frac{\chi^{\lambda}(D(q))}{\chi^{[1]}(D(q))^{n}} \\
& =\left(\frac{\sin (\pi / l)}{\sin (k \pi / l)}\right)^{n} \prod_{1 \leq r<s \leq k} \frac{\sin \left\{\left(\lambda_{r}-\lambda_{s}+s-r\right) \pi / l\right\}}{\sin \{(s-r) \pi / l\}},
\end{aligned}
$$

where $\chi^{\lambda}(D(q))$ is the character corresponding to the representation of $S U(k)$ given by $\lambda$, evaluated at the diagonal matrix $D(q)=\operatorname{diag}\left\{q^{\frac{k+1}{2}-i}, i=1, \ldots, k\right\}$. The $w_{\lambda}$ 's are also specializations of the Schur functions $s_{\lambda}$.

For each $q=e^{ \pm 2 \pi i / l}$, for $1<k<l, l \geq 3$, and for each $m \in \mathbb{N}_{0}$ we have a pair of $\mathrm{II}_{1}$ factors $C \subseteq D$. By (3.3.2), the index for the pair $C \subseteq D$ is

$$
[D: C]=\left(W^{n}\left\|\vec{t}^{(n)}\right\|^{2}\right) W^{m},
$$

for $n>n_{0}$, where $\vec{t}^{(n)}$ is the weight vector for the trace on $A_{n}$, and $W$ is the index for the one-sided pair $\left\langle g_{2}, \ldots, g_{n}, \ldots\right\rangle^{\prime \prime} \subseteq\left\langle g_{1}, \ldots, g_{n}, \ldots\right\rangle^{\prime \prime}$. This value was computed in $[\mathrm{W}-1]$. For $r>n_{0}$,

$$
W=\frac{\left\|\vec{t}^{(r)}\right\|^{2}}{\left\|\vec{t}^{(r+1)}\right\|^{2}}=\frac{\sin ^{2}(k \pi / l)}{\sin ^{2}(\pi / l)}
$$

Then, for large $n$

$$
[D: C]=\left\|\vec{t}^{(n)}\right\|^{2}\left(\frac{\sin ^{2}(k \pi / l)}{\sin ^{2}(\pi / l)}\right)^{n+m} .
$$

So, it remains to compute $\left\|\vec{t}^{(n)}\right\|^{2}$ for large $n$. Using the expression for the weight vector for the trace in (4.2.1), we get

$$
\left\|\vec{t}^{(n)}\right\|^{2}=\left(\frac{\sin ^{2}(\pi / l)}{\sin ^{2}(k \pi / l)}\right)^{n} \sum_{\lambda \in \Lambda_{n}^{(k, l)}} \prod_{1 \leq r<s \leq k} \frac{\sin ^{2}\left\{\left(\lambda_{r}-\lambda_{s}+s-r\right) \pi / l\right\}}{\sin ^{2}\{(s-r) \pi / l\}} .
$$

Since the expression for $[D: C]$ above holds for every $n>n_{0}$ by Wenzl's formula, in particular it will hold for $n+i$, with $i=0, \ldots, k-1$, if $n>n_{0}$. Combined with 
the expression for $\left\|\vec{t}^{(n)}\right\|^{2}$ above, this gives us

$$
[D: C]=\left(\frac{\sin (k \pi / l)}{\sin (\pi / l)}\right)^{2 m} \frac{1}{k} \sum_{i=0}^{k-1} \sum_{\lambda \in \Lambda_{n+i}^{(k, l)}} \prod_{1 \leq r<s \leq k} \frac{\sin ^{2}\left\{\left(\lambda_{r}-\lambda_{s}+s-r\right) \pi / l\right\}}{\sin ^{2}\{(s-r) \pi / l\}} .
$$

To continue we shall need some lemmas:

Lemma 4.2.1. For $n \geq(l-k)(k-1)$ there is a bijection between $\bigcup_{j=0}^{k-1} \Lambda_{n+j}^{(k, l)}$ and the set $\left\{\left(r_{1}, r_{2}, \ldots, r_{k-1}, 0\right)\right.$ such that $\left.l>r_{1}>r_{2}>\ldots>r_{k-1}>0\right\}$.

Proof. Define $\psi$ on $\bigcup_{j=0}^{k-1} \Lambda_{n+j}^{(k, l)}$ by $\lambda \mapsto r$, with $r_{i}=\lambda_{i}-\lambda_{k}+k-i$, where $\lambda=$ $\left(\lambda_{1}, \lambda_{2}, \ldots, \lambda_{k}\right), r=\left(r_{1}, r_{2}, \ldots, r_{k-1}, 0\right)$. If $\psi(\lambda)=\psi(\tilde{\lambda})$, then $\lambda_{i}-\lambda_{k}=\tilde{\lambda}_{i}-\tilde{\lambda}_{k}$, for all $i=1, \ldots, k$, and so $\sum_{i=1}^{k} \lambda_{i}-k \lambda_{k}=\sum_{i=1}^{k} \tilde{\lambda}_{i}-k \tilde{\lambda}_{k}$. If $\lambda \in \Lambda_{n+s}^{(k, l)}$ and $\tilde{\lambda} \in \Lambda_{n+t}^{(k, l)}$, then we have that $n+s-k \lambda_{k}=n+t-k \tilde{\lambda}_{k}$, and so, $k\left(\lambda_{k}-\tilde{\lambda}_{k}\right)=s-t$. Since $0 \leq s, t \leq k-1$, and $k$ divides $(s-t)$, then $s-t=0=\lambda_{k}-\tilde{\lambda}_{k}$. Therefore, $\lambda=\tilde{\lambda}$, and $\psi$ is injective. Given $r=\left(r_{1}, r_{2}, \ldots, r_{k-1}, 0\right)$ such that $l>r_{1}>r_{2}>\ldots .>r_{k-1}>0$, there exist a unique $s \in \mathbb{N}, 0 \leq s \leq k-1$, and a unique $p \in \mathbb{N}_{0}$ such that $n+\frac{k(k-1)}{2}-\sum_{i=1}^{k-1} r_{i}=p k-s$. If $\lambda \in \bigcup_{j=0}^{k-1} \Lambda_{n+j}^{(k, l)}$ is given by $\lambda_{i}=r_{i}+p-k+i$ for $i=1, \ldots, k$, then $\psi(\lambda)=r$ and surjectivity holds.

By applying Lemma 4.2.1 we obtain that

$$
\begin{aligned}
I: & =\sum_{i=0}^{k-1} \sum_{\lambda \in \Lambda_{n+i}^{(k, l)}} \prod_{1 \leq r<s \leq k} \sin ^{2}\left\{\left(\lambda_{r}-\lambda_{s}+s-r\right) \pi / l\right\} \\
& =\sum_{l>l_{1}>\ldots>l_{k-1}>0} \prod_{1 \leq i<j \leq k} \sin ^{2}\left\{\left(l_{i}-l_{j}\right) \pi / l\right\} .
\end{aligned}
$$

Since we have that $\sin ^{2}\left\{\left(l_{i}-l_{i}\right) \pi / l\right\}=0, \sin ^{2}\left\{\left(l-l_{k}\right) \pi / l\right\}=0$ (recall that $l_{k}=0$ ), and $\sin ^{2}\left\{\left(l_{i}-l_{j}\right) \pi / l\right\}=\sin ^{2}\left\{\left(l_{j}-l_{i}\right) \pi / l\right\}$ for all $i$ and $j$, then

$$
\begin{aligned}
I & =\sum_{l \geq l_{1} \geq \ldots \geq l_{k-1}>0} \prod_{1 \leq i<j \leq k} \sin ^{2}\left\{\left(l_{i}-l_{j}\right) \pi / l\right\} \\
& =\frac{1}{(k-1) !} \sum_{l_{1}, \ldots, l_{k-1}=1}^{l} \prod_{1 \leq i<j \leq k} \sin ^{2}\left\{\left(l_{i}-l_{j}\right) \pi / l\right\} \\
& =\frac{1}{(k-1) ! 2^{k(k-1)}} \sum_{l_{1}, \ldots . l_{k-1}=1}^{l}\left(\prod_{1 \leq i<j \leq k}\left(q^{l_{i}}-q^{l_{j}}\right)\right)\left(\prod_{1 \leq i<j \leq k}\left(q^{-l_{i}}-q^{-l_{j}}\right)\right)
\end{aligned}
$$

by setting $q=e^{ \pm 2 \pi i / l}$, and writing $\sin ^{2}\left\{\left(l_{i}-l_{j}\right) \pi / l\right\}$ in terms of $q$. Note that the factors in the summation above have the form of determinants of Vandermonde matrices. Recall that a $k \times k$ Vandermonde matrix is a matrix $X$ of the form

$$
X=\left(\begin{array}{cccc}
1 & 1 & \ldots & 1 \\
x_{1} & x_{2} & \ldots & x_{k} \\
x_{1}^{2} & x_{2}^{2} & \ldots & x_{k}^{2} \\
\vdots & \vdots & \ddots & \vdots \\
x_{1}^{k-1} & x_{2}^{k-1} & \ldots & x_{k}^{k-1}
\end{array}\right)
$$


where the $x_{i}$ 's are variables. The determinant of the Vandermonde matrix $X$ is given by $\operatorname{det} X=\prod_{1 \leq i<j \leq k}\left(x_{i}-x_{j}\right)$. Let $x_{i}$ and $y_{i}$ be given by $x_{i}=q^{l_{i}}$ and $y_{i}=q^{-l_{i}}$ for $i=1, \ldots, k-1$, and let $X$ and $Y$ be the respective Vandermonde matrices. Rewriting $I$, we have

$$
I=\frac{1}{(k-1) ! 2^{k(k-1)}} \sum_{l_{1}, \ldots, l_{k-1}=1}^{l} \operatorname{det}(X) \operatorname{det}(Y) .
$$

Lemma 4.2.2.

$$
\sum_{l_{1}, \ldots, l_{k-1}=1}^{l} \operatorname{det}(X) \operatorname{det}(Y)=l^{k-1} k ! .
$$

Proof. We have that $\sum_{l_{i}=1}^{l} y_{i}^{j} x_{i}^{s}=\delta_{j, s} l$, for $i=1, \ldots, k$ (recall that $q=e^{ \pm 2 \pi i / l}$ ). Thus, if we sum over one variable at a time we have $\sum_{l_{1}, \ldots, l_{k-1}=1}^{l} \operatorname{det}(X) \operatorname{det}(Y)=$ $l^{k-1} c$, where $c$ is the constant term of the Laurent polynomial $\operatorname{det}(X) \operatorname{det}(Y)$. By definition, $\operatorname{det}(X) \operatorname{det}(Y)=\sum_{\sigma, \nu \in S_{k}} s g(\nu) s g(\sigma) x_{\sigma(2)}^{1} \ldots x_{\sigma(k)}^{k-1} y_{\nu(2)}^{1} \ldots y_{\nu(k)}^{k-1}$, and one can see that the constant term appears only when $\nu=\sigma$. So, $c=\sum_{\sigma \in S_{k}} 1=k$ !.

Theorem 4.2.3. (i) If $C \subseteq D$ is the pair of the $\mathrm{II}_{1}$ factors defined as before, then the index $[D: C]$ is given by

$$
\frac{l^{(k-1)}}{2^{k(k-1)}} \frac{\sin ^{2 m}(k \pi / l)}{\sin ^{2 m}(\pi / l)} \frac{1}{\prod_{1 \leq r<s \leq k} \sin ^{2}\{(r-s) \pi / l\}} .
$$

(ii) For each $(k, l)$ diagram $\lambda$ of size $m$, there exists a subfactor of the hyperfinite $\mathrm{II}_{1}$ factor with trivial relative commutant and index $\left[D_{\lambda}: C_{\lambda}\right]$ given by

$$
\frac{l^{k-1}}{2^{k(k-1)}} \prod_{1 \leq r<s \leq k} \frac{\sin ^{2}\left\{\left(\lambda_{r}-\lambda_{s}+s-r\right) \pi / l\right\}}{\sin ^{4}\{(s-r) \pi / l\}} .
$$

Proof. (i) By (4.2.4) and Lemma 4.2.1 we have that $I=\frac{l^{k-1} k}{2^{k(k-1)}}$. Then, by (4.2.2) and (4.2.3), we obtain the desired formula.

(ii) Let $m$ be a non-negative integer, and let $\lambda$ be in $\Lambda_{m}^{(k, l)}$. By section 3.4, the relative commutant for the pair $C \subseteq D$ (obtained after fixing $m$ ) is

$$
C^{\prime} \cap D=\left\langle g_{1}, \ldots, g_{m-1}\right\rangle=A_{m} \cong \bigoplus_{\lambda \in \Lambda_{m}^{(k, l)}} M_{a_{\lambda}}(\mathbb{C}) .
$$

If $p$ is a minimal idempotent of $C^{\prime} \cap D$, the reduced pair of factors $C_{p} \subseteq D_{p}$ is an irreducible pair. Let us consider $p=p_{\lambda} \in C_{p}^{\prime} \cap D_{p}$ a minimal idempotent labeled by $\lambda$ in $\Lambda_{m}^{(k, l)}$. Using (4.2.1) and [W-1, Theorem 1.5, (iii)], we have the desired expression.

4.3 Subfactors of types B,C,D: The algebra $C_{\infty}(r, q)$. The algebras $C_{f}(r, q)$ in two complex parameters $r$ and $q$ (the so-called Birman-Murakami-Wenzl algebras) were first derived from the Kauffman link invariant (see [W-2], [BW], [M]). Roughly, since one can obtain a link by 'closing' a braid, link invariants may induce trace functionals on the braid group with the Markov property. The GNS construction with respect to the trace derived from the Kauffman link invariant gives the definition of the algebra $C_{\infty}(r, q)$. See [W-2] for details on this construction. 
Algebraically, the complex algebra $C_{f}(r, q)$ is given by generators $1, T_{1}, \ldots, T_{f-1}$, which are assumed to be invertible, and relations

(B) $T_{i+1} T_{i} T_{i+1}=T_{i} T_{i+1} T_{i}$, for $i=1, \ldots, n-2$,

(B) $T_{i} T_{j}=T_{j} T_{i}$, for $|i-j| \geq 2$,

$\left(R_{1}\right) e_{i} T_{i}=r^{-1} e_{i}$, for $1 \leq i \leq f-1$,

$\left(R_{2}\right) e_{i} T_{i}^{ \pm 1} e_{i}=r^{ \pm 1} e_{i}$, for $1 \leq i \leq f-1$,

where for $q \neq 0, \pm 1, e_{i}$ is defined by the equation

$$
\left(q-q^{-1}\right)\left(1-e_{i}\right)=T_{i}-T_{i}^{-1},
$$

and it is also a multiple of the characteristic idempotent $p_{i}$ belonging to the characteristic value $r^{-1}$ of $T_{i}$. The other characteristic values of $T_{i}$ are $q$ and $-q^{-1}$.

$\left(R_{1}\right)$ can be replaced by $\left(R_{1}\right)^{\prime}$ :

$$
\left(T_{i}-r^{-1}\right)\left(T_{i}+q^{-1}\right)\left(T_{i}-q\right)=0 .
$$

A different set of relations can also be given so that the algebra is also well defined at $(r, q)=(1,1)$ (see [W-2, page 401]).

These algebras are related to the Brauer algebras in the following sense. The algebra $C_{\infty}(r, q)=\bigcup C_{f}(r, q)$ can be described by adding to the two dimensional description of the braid group $\mathbf{B}_{f}$ additional generators $\epsilon_{i}$ ("horizontal" strands) as shown e.g. in $[\mathrm{W}-2, \S 3]$. Since multiplication is by concatenation of the diagrams, one should get rid of the resulting circles by multiplying the graph without the circles by an expression depending on $r, q$, and the number of circles.

One can define inductively a functional $t r$ on the $C_{f}(r, q)$ 's which turns out to be a trace, and it is actually the one derived from the the Kauffman invariant; it also agrees with the trace obtained via the quantum group approach outlined in section 4.1, see [W-2, Lemma 3.4]. We consider the GNS construction $\pi_{t r}$ with respect to the trace (the quotient modulo the anhihilator ideal).

In [W-2, Lemma 4.1] it is proven inductively that the quotient $\pi_{t r}\left(C_{f}(r, q)\right)$ is semisimple if certain quotients of the Hecke algebra, $K_{s}:=\rho_{s}\left(H_{s}\left(q^{2}\right)\right)$, are also semisimple for $s=1, \ldots, f$. Furthermore, it says that in this case the structure of the $\pi_{t r}\left(C_{f}(r, q)\right)$ 's is completely determined if one knows the representations $\rho_{f}$ of $H_{f}\left(q^{2}\right)$ for all $f \in \mathbb{N}$. Also, in this case the structure can be encoded in the graph $\Gamma(r, q)$ whose vertices at the $f^{t h}$ level are labelled by the simple components of $K_{f}$, and whose edges are determined by the decomposition of an irreducible $K_{f}$ module into irreducible $K_{f-1}$ modules. The representations $\rho_{f}$ of $H_{f}\left(q^{2}\right)$ are direct sums of the representations $\pi_{\lambda}^{(k, l)}$ when $q^{2}$ is of the form $e^{ \pm 2 \pi i / l}$ (as mentioned in the last section), or of the representations $\pi_{\lambda}$, when $q^{2}$ is not a root of unity (see [W-1]). In particular, the vertices of $\Gamma(r, q)$ are labelled by Young diagrams and $\Gamma(r, q)$ is a subgraph of the Young lattice.

In the semisimple case, Wenzl's lemma also tells us that $\Gamma(r, q)$ determines the Bratteli diagram for $\ldots \subseteq \pi_{t r}\left(C_{f-1}(r, q)\right) \subseteq \pi_{t r}\left(C_{f}(r, q)\right) \subseteq \ldots$ as follows: The diagram at the $(f-1)^{s t}$ to $f^{\text {th }}$ level is given by the 'reflection' of the diagram at the $(f-2)^{n d}$ to $(f-1)^{s t}$ level plus a 'new part' obtained by joining the Young diagrams of size $(f-1)$ in $\Gamma(r, q)$ with the ones of size $f$. Thus, the labels for the simple ideals of $\pi_{t r}\left(C_{f}(r, q)\right)$ are given by $\Gamma_{f}(r, q):=\{\lambda \in \Gamma(r, q):|\lambda|=f, f-2, \ldots, 1$ or 0$\}$, and furthermore, a vertex labelled by $\lambda$ at the level $(f-1)$ on the Bratteli diagram is connected to a vertex labelled by $\mu$ at level $f$ if and only if $\mu$ is obtained from $\lambda$ by adding or removing one box. 
If $\Gamma(r, q)$ is finite then the sequence $\left(\pi_{t r}\left(C_{f}(r, q)\right)\right)_{f}$ has periodicity 2. The difficulty is to determine which diagrams appear in $\Gamma(r, q)$. This was done by Wenzl in [W-2, Theorem 6.4], where he finds the values for $r$ and $q$ for which these quotients are semisimple and the representations $\pi_{t r}$ unitary, together with a description of the corresponding graphs $\Gamma(r, q)$. We shall write explicitly these graphs $\Gamma(r, q)$ for each Lie type while computing the subfactors' indices in the next sections. The values for $r$ and $q$ are of the form $q=e^{\pi i / l}$ and $r=q^{n}$, with $l \in \mathbb{N}$ and $|n|<l, n \neq-1$. Moreover, for these values the representations $\pi_{t r}$ satisfy the conditions (i)-(v) of section 3.1 .

Set

$$
x=\frac{r-r^{-1}}{q-q^{-1}}+1 .
$$

By [W-2, Theorem 4.6], the weight vector for the Markov trace $\operatorname{tr}$ for $\pi_{t r}\left(C_{f}(r, q)\right)$ is given by

$$
\left(Q_{\lambda}(r, q) / x^{f}\right)_{\Gamma_{f}(r, q)}
$$

where $Q_{\lambda}$ is a non-zero rational function independent of $f$. There is the possible exception of the diagram $\lambda$ given by the hook $\lambda=\left[l-k+1,1^{k-1}\right]$, in which case one should take $\tilde{Q}_{\lambda}(r, q)=\left(Q_{\lambda}+Q_{\left[l-k+2,1^{k-2}\right]}\right)(r, q)$. We have, by [W-2, Theorem $5.5]$,

$$
Q_{\lambda}(r, q)=\prod_{(j, j) \in \lambda} \frac{\left[y+\lambda_{j}-\lambda_{j}^{\prime}\right]_{q}+[h(j, j)]_{q}}{[h(j, j)]_{q}} \prod_{(i, j) \in \lambda, i \neq j} \frac{[y+d(i, j)]_{q}}{[h(i, j)]_{q}},
$$

where $[s]_{q}:=q^{s}-q^{-s},[y+s]_{q}:=r q^{s}-r^{-1} q^{-s}, \lambda_{i}^{\prime}$ is the length of the $i^{\text {th }}$ column of $\lambda, h(i, j):=\lambda_{i}-i+\lambda_{j}^{\prime}-j+1$ (the hook length), and $d(i, j):=\lambda_{i}+\lambda_{j}-i-j+1$ if $i \leq j$, and $d(i, j):=-\lambda_{i}^{\prime}-\lambda_{j}^{\prime}+i+j-1$ if $i>j$.

As mentioned in section 4.1, the weight vector in (4.3.2) coincides with

$$
\left(\frac{\chi^{(\tilde{\lambda})}(D(q))}{\operatorname{Tr}(D(q))^{f}}\right)_{\lambda \in \Gamma_{f}(r, q)} .
$$

Here, $\chi^{(\tilde{\lambda})}$ is the character corresponding to an irreducible representation $\varrho_{\tilde{\lambda}}$ of the Lie group which appears in the $f$-fold tensor power of the standard representation. The diagram $\tilde{\lambda}$ is obtained from $\lambda$ by a rule depending on the Lie type: For the even or odd orthogonal types $O(n), \tilde{\lambda}=\lambda$ if $\lambda_{1}^{\prime} \leq\left[\frac{n}{2}\right]$, and otherwise it is given by $\tilde{\lambda}_{1}^{\prime}:=n-\lambda_{1}^{\prime}$ and $\tilde{\lambda}_{i}^{\prime}:=\lambda_{i}^{\prime}$ for $i \geq 2$. For the symplectic case $\operatorname{Sp}(2 k), \tilde{\lambda}:=\lambda^{\prime}$, the 'transpose' of $\lambda$. The diagonal matrix $D(q)$ is given by $\operatorname{diag}\left\{q^{i}: i \in I\right\}$, where $I$ is the set correspoding to the eigenvalues of a matrix given by the sum of the positive roots of $\mathcal{G}$ with respect to a suitably normalized invariant bilinear form. Note that for the B,C,D types we do not consider the half sum of positive roots as in the type A case, but the whole sum: This is to be consistent with Wenzl's choice of $q$ in [W-2, Theorem 6.4] (if we considered the half sum we woud need to work with $q^{2}$ instead).

Taking the values for the parameters $r$ and $q$ as above, we can define the pairs of hyperfinite $\mathrm{II}_{1}$ factors $C \subseteq D$ and proceed with their indices. By (3.3.2) and for 
large $f$,

$$
[D: C]=\left\|\vec{t}^{(f)}\right\|^{2} W^{(f+m)},
$$

where $\vec{t}^{(f)}$ is the weight vector for the trace on $A_{f}=\pi_{t r}\left(C_{f}(r, q)\right)=\left\langle g_{1}, \ldots, g_{f-1}\right\rangle$, and $W$ the index for the one-sided pair $\left\langle g_{2}, \ldots, g_{s}, \ldots\right\rangle^{\prime \prime} \subseteq\left\langle g_{1}, \ldots, g_{s}, \ldots\right\rangle^{\prime \prime}$, which was computed in [W-2, Theorem 7.1] and for large $n$ is equal to

$$
W=\frac{\left\|\vec{t}^{(n)}\right\|^{2}}{\left\|\vec{t}^{(n+1)}\right\|^{2}}=x^{2},
$$

where, as in (4.3.1), $x=\frac{r-r^{-1}}{q-q^{-1}}+1=\sum_{i \in I} q^{i}$. Then, for large $f$

$$
\begin{aligned}
{[D: C] } & =x^{2(f+m)}\left\|\vec{t}^{(f)}\right\|^{2}=x^{2 m} \sum_{\lambda \in \Gamma_{f}(r, q)} Q_{\lambda}^{2}(r, q) \\
& =\frac{1}{2} x^{2 m} \sum_{\lambda \in \Gamma(r, q)} Q_{\lambda}^{2}(r, q),
\end{aligned}
$$

by (4.3.2) and because $\Gamma_{f}(r, q)=\{\lambda \in \Gamma(r, q):|\lambda|=f, f-2, \ldots, 1$ or 0$\}$.

As mentioned in the introduction, for $m=0$, our two-sided subfactors corresponding to the unitary braid representations associated to the Lie types B,C,D coincide with the asymptotic inclusions for their one-sided versions or Wenzl subfactors. So, before continuing with the computation of our indices we shall prove this fact.

The asymptotic inclusion for a pair of $\mathrm{II}_{1}$ subfactors $N \subseteq M$ (see $[\mathrm{O}]$ ) is defined by the pair given by

$$
N \vee\left(N^{\prime} \cap M^{(\infty)}\right) \subseteq M^{(\infty)},
$$

where $M^{(\infty)}:=\left(\bigcup_{i} M^{(i)}\right)^{- \text {w.o. }}$ and $M^{(i)}$ is the Jones' $i^{\text {th }}$ basic construction.

Consider now the one-sided pair $N:=\left\langle g_{1}, g_{2}, \ldots\right\rangle^{\prime \prime} \subseteq M:=\left\langle g_{0}, g_{1}, g_{2}, \ldots\right\rangle^{\prime \prime}$ and the two-sided pair $C:=\left\langle\ldots g_{-1}, g_{1}, \ldots\right\rangle^{\prime \prime} \subseteq D:=\left\langle\ldots g_{-1}, g_{0}, g_{1}, \ldots\right\rangle^{\prime \prime}$. Note that in both cases the subfactors are obtained by leaving out one braid generator, $g_{0}$. Wenzl proved in $[\mathrm{W}-2, \S 7]$ that for his one-sided type B,C,D subfactors $N \subseteq M$, and for every natural number $i$, there is an isomorphism

$$
\Psi^{(i)}: M^{(i)}=\left\langle\bar{e}_{i}, \bar{e}_{i-1}, \ldots, \bar{e}_{1}, g_{0}, g_{1}, \ldots\right\rangle^{\prime \prime} \rightarrow\left\langle g_{0}, g_{1}, \ldots\right\rangle^{\prime \prime},
$$

mapping $N=\left\langle g_{1}, \ldots\right\rangle^{\prime \prime}$ onto $\left\langle g_{i}, g_{i+1}, \ldots\right\rangle^{\prime \prime}$, and where $\bar{e}_{j}$ is the Jones' $j^{\text {th }}$ basic projection for $N \subseteq M$. This map takes the projections $\bar{e}_{j}$ to the projections $\rho\left(e_{j-1}\right)$, where $e_{j}$ is a multiple of the spectral projection $p_{j}$ corresponding to the characteristic eigenvalue $r^{-1}$ of $T_{j}$ (see defining relations for the algebra $C_{\infty}(r, q)$ at the beginning of this section). As a corollary, from this he obtains that the higher relative commutant $N^{\prime} \cap M^{(i)}$ for $N \subseteq M$ is isomorphic to the algebra $\left\langle g_{0}, \ldots, g_{i-2}\right\rangle$.

If we compose the isomorphism $\Psi^{(i)}$ with an isomorphism given by a relabelling of the generators, shift $-i+1$, we then have an isomorphism

$$
\phi_{i}: M^{(i)}=\left\langle\bar{e}_{i}, \bar{e}_{i-1}, \ldots, \bar{e}_{1}, g_{0}, g_{1}, \ldots\right\rangle^{\prime \prime} \rightarrow\left\langle g_{-i+1}, g_{-i+2}, \ldots, g_{0}, g_{1}, \ldots\right\rangle^{\prime \prime},
$$

mapping $N=\left\langle g_{1}, \ldots\right\rangle^{\prime \prime}$ onto $\left\langle g_{1}, \ldots\right\rangle^{\prime \prime}$. Therefore, for all $i \in \mathbb{N}$ we have that

$$
N^{\prime} \cap M^{(i)} \cong_{\phi_{i}}\left\langle g_{-i+1}, g_{-i+2}, \ldots, g_{-1}\right\rangle .
$$

Note that the $\phi_{i}$ 's are coherent, so that we can define an isomorphism $\Phi: M^{(\infty)} \rightarrow$ $D$, with $\Phi\left(N \vee\left(N^{\prime} \cap M^{(\infty)}\right)\right)=C$. We have proved 
Proposition 4.3.3. The pair of subfactors $C \subseteq D$ associated to the Lie types $B, C, D$, as above, is conjugate to the asymptotic pair corresponding to the one-sided versions (the Wenzl subfactors) $N=\left\langle g_{1}, g_{2}, \ldots\right\rangle^{\prime \prime} \subseteq M=\left\langle g_{0}, g_{1}, g_{2}, \ldots\right\rangle^{\prime \prime}$, that is,

$$
\left(N \vee\left(N^{\prime} \cap M^{(\infty)}\right) \subseteq M^{(\infty)}\right) \simeq(C \subseteq D)
$$

4.4 Index for subfactors of type B: The odd orthogonal case $O(2 k+1)$. This is the case where the values for $r$ and $q$ are given by $q=e^{\pi i / l}$ and $r=q^{2 k}$, with $2<2 k<l-2$ and $l>6$. We have, by [W-2, Theorem 6.4, (a)],

$$
\begin{gathered}
\Gamma\left(q^{2 k}, q\right)=\left\{\lambda: \lambda_{1}+\lambda_{2} \leq l-2 k+1 \text { and } \lambda_{1}^{\prime}+\lambda_{2}^{\prime} \leq 2 k+1\right\} \\
\cup\left\{\left[l-2 k+1,1^{2 k-1}\right]\right\} .
\end{gathered}
$$

Lemma 4.4.1. If $\lambda \in \Gamma\left(q^{2 k}, q\right)$, and $\lambda_{1}^{\prime}>k$, then there exists $\psi(\lambda)=\tilde{\lambda} \in \Gamma\left(q^{2 k}, q\right)$ such that $\tilde{\lambda}_{1}^{\prime} \leq k$ and such that $Q_{\lambda}\left(q^{2 k}, q\right)=Q_{\tilde{\lambda}}\left(q^{2 k}, q\right)$. Furthermore, the map $\psi$ is a bijection from $\Gamma\left(q^{2 k}, q\right) \cap\left\{\lambda: \lambda_{1}^{\prime}>k\right\}$ onto $\Gamma\left(q^{2 k}, q\right) \cap\left\{\lambda: \lambda_{1}^{\prime} \leq k\right\}$.

Proof. Define $\psi(\lambda)=\tilde{\lambda}$ by $\tilde{\lambda}_{i}^{\prime}=\lambda_{i}^{\prime}$ for $i=2, \ldots, \lambda_{1}$ and $\tilde{\lambda}_{1}^{\prime}=2 k+1-\lambda_{1}^{\prime}$. We have that $\lambda_{1}^{\prime}>k$ and $\lambda_{1}^{\prime}+\lambda_{2}^{\prime} \leq 2 k+1$ give $\tilde{\lambda}_{2}^{\prime} \leq \tilde{\lambda}_{1}^{\prime}$ (so that $\tilde{\lambda}$ is a Young diagram), $\tilde{\lambda}_{1}^{\prime} \leq k$ and $\tilde{\lambda}_{1}^{\prime}+\tilde{\lambda}_{2}^{\prime} \leq 2 k+1$. Also, $\tilde{\lambda}_{1}+\tilde{\lambda}_{2}=\lambda_{1}+\tilde{\lambda}_{2} \leq \lambda_{1}+\lambda_{2} \leq l-2 k+1$. Therefore, $\tilde{\lambda} \in \Gamma\left(q^{2 k}, q\right)$ and $\tilde{\lambda}_{1}^{\prime} \leq k$. Similarly, the inverse map is well defined: For $\tilde{\lambda} \in \Gamma\left(q^{2 k}, q\right)$ with $\tilde{\lambda}_{1}^{\prime} \leq k$, take $\lambda$ with $\lambda_{i}^{\prime}=\tilde{\lambda}_{i}^{\prime}$ for $i=2, \ldots, \tilde{\lambda}_{1}$ and $\lambda_{1}^{\prime}=2 k+1-\tilde{\lambda}_{1}^{\prime}$. We have that $\lambda_{1}^{\prime} \geq \lambda_{2}^{\prime}$ and $\lambda_{1}^{\prime}>k$. If $\tilde{\lambda}$ is not just one row (that is, if $\tilde{\lambda}_{1}^{\prime}>1$ ), then $\lambda_{1}+\lambda_{2} \leq l-2 k+1$. If $\tilde{\lambda}_{1}^{\prime}=1$, then either $\lambda$ is the hook $\left[l-2 k+1,1^{2 k-1}\right]$ (when $\tilde{\lambda}=[l-2 k+1]$ ), or we have $\lambda_{1}+\lambda_{2} \leq l-2 k+1$ (when $\tilde{\lambda}=\left[\tilde{\lambda}_{1}\right]$ with $\left.\tilde{\lambda}_{1}<l-2 k+1\right)$. The fact that $Q_{\lambda}\left(q^{2 k}, q\right)=Q_{\tilde{\lambda}}\left(q^{2 k}, q\right)$ follows from (4.3.3).

For $\lambda \in \Gamma\left(q^{2 k}, q\right)$ with $\lambda_{1}^{\prime} \leq k$, we have that $Q_{\lambda}\left(q^{2 k}, q\right)=\chi^{(\lambda)}(D(q))$, where $D(q) \in O(2 k+1)$ is the diagonal matrix with eigenvalues $\left\{1, q^{ \pm(2 i-1)}, i=1, \ldots, k\right\}$, by (4.3.4). The character formula for the representation of $O(2 k+1)$ given by $\lambda=\left(\lambda_{1}, \ldots, \lambda_{k}\right)$ is the following (see [Mi], [We], [FH, Ch.24], or [W-2]):

$$
\chi^{(\lambda)}(D(q))=\tilde{\chi}^{(\lambda)}(D(q))=\frac{\operatorname{det}\left(\left(q^{(2 i-1) \alpha_{j}}-q^{-(2 i-1) \alpha_{j}}\right)_{i, j}\right)}{\operatorname{det}\left(\left(q^{(2 i-1) \alpha_{j}^{0}}-q^{-(2 i-1) \alpha_{j}^{0}}\right)_{i, j}\right)},
$$

where $\alpha_{j}:=\lambda_{j}+k-j+\frac{1}{2}$ and $\alpha_{j}^{0}:=k-j+\frac{1}{2}$, and where $\tilde{\chi}^{(\lambda)}$ is the character of the restriction of $\lambda$ to $S O(2 k+1)$. We can rewrite the formula as

$$
\chi^{(\lambda)}(D(q))=\prod_{s=1}^{k} \frac{\left[l_{s}-\frac{1}{2}\right]_{q}}{\left[l_{s}^{0}-\frac{1}{2}\right]_{q}} \prod_{1 \leq s<t \leq k} \frac{\left[l_{s}+l_{t}-1\right]_{q}\left[l_{s}-l_{t}\right]_{q}}{\left[l_{s}^{0}+l_{t}^{0}-1\right]_{q}\left[l_{s}^{0}-l_{t}^{0}\right]_{q}},
$$

where $l_{j}:=\alpha_{j}+\frac{1}{2}=\lambda_{j}+k-j+1$ and $l_{j}^{0}:=\alpha_{j}^{0}+\frac{1}{2}=k-j+1$. Define

$$
x_{s}=\sin ^{2}\left(\frac{\left(l_{s}-\frac{1}{2}\right) \pi}{l}\right) .
$$


By (4.3.5) we need to compute $Q:=\sum_{\lambda \in \Gamma\left(q^{2 k}, q\right)} Q_{\lambda}^{2}\left(q^{2 k}, q\right)$. By Lemma 4.4.1, (4.4.1) and (4.4.2) we have

$$
\begin{aligned}
Q & =2 \sum_{\left\{\lambda \in \Gamma\left(q^{2 k}, q\right): \lambda_{1}^{\prime} \leq k\right\}} Q_{\lambda}^{2}\left(q^{2 k}, q\right) \\
& =\frac{2 \sum_{\left\{\lambda \in \Gamma\left(q^{2 k}, q\right): \lambda_{1}^{\prime} \leq k\right\}} \prod_{s=1}^{k}\left[l_{s}-\frac{1}{2}\right]_{q}^{2} \prod_{1 \leq s<t \leq k}\left[l_{s}+l_{t}-1\right]_{q}^{2}\left[l_{s}-l_{t}\right]_{q}^{2}}{\prod_{s=1}^{k}\left[l_{s}^{0}-\frac{1}{2}\right]_{q}^{2} \prod_{1 \leq s<t \leq k}\left[l_{s}^{0}+l_{t}^{0}-1\right]_{q}^{2}\left[l_{s}^{0}-l_{t}^{0}\right]_{q}^{2}} \\
& =\frac{2 \sum_{\left\{\lambda \in \Gamma\left(q^{2 k}, q\right): \lambda_{1}^{\prime} \leq k\right\}} \prod_{s=1}^{k} x_{s} \prod_{1 \leq s<t \leq k}\left(x_{s}-x_{t}\right)^{2}}{\prod_{s=1}^{k} \sin ^{2}\left(\left(s+\frac{1}{2}\right) \pi / l\right) \prod_{1 \leq s<t \leq k} \sin ^{2}((t+s+1) \pi / l) \sin ^{2}((t-s) \pi / l)},
\end{aligned}
$$

where we use the identity $[a-b]_{q}^{2}[a+b]_{q}^{2}=16\left(\sin ^{2}\left(\frac{a \pi}{l}\right)-\sin ^{2}\left(\frac{b \pi}{l}\right)\right)^{2}$.

Lemma 4.4.2. (i) Set $T_{1}=\left\{\lambda \in \Gamma\left(q^{2 k}, q\right): \lambda_{1}^{\prime} \leq k\right\}$. The map $\phi: T_{1} \rightarrow T_{2}:=$ $\left\{h=\left(l_{1}, \ldots, l_{k}\right): l_{1}+l_{2} \leq l\right.$ and $\left.l_{1}>l_{2}>\ldots>l_{k}>0\right\}$ is a bijection, where $\lambda \mapsto \lambda+(k, k-1, \ldots, 1)$.

(ii) Set $P=\prod_{s=1}^{k} x_{s} \prod_{1 \leq s<t \leq k}\left(x_{s}-x_{t}\right)^{2}$. Then

$$
\sum_{\lambda \in T_{1}} P=\sum_{h \in T_{2}} P=\sum_{h \in T_{3}} P
$$

where $T_{3}=\left\{h=\left(l_{1}, \ldots, l_{k}\right): l \geq l_{1} \geq \ldots \geq l_{k} \geq 1, l_{1}+l_{2} \leq l+1\right\}$.

$$
\sum_{h \in T_{3}} P=\frac{1}{2^{k-1} k !} \sum_{l_{1}, \ldots, l_{k}=1}^{l} P=\frac{1}{2^{k-1}} \sum_{h \in T} P,
$$

where $T=\left\{h=\left(l_{1}, \ldots, l_{k}\right): l \geq l_{1} \geq \ldots \geq l_{k} \geq 1\right\}$.

Proof. (i) Evident.

(ii) For the second equality in (4.4.4), note that if $h=\left(l_{1}, \ldots, l_{k}\right)$ has $l_{i}=l_{j}$ for $i \neq j$ then $P=0$. Also, if $l_{1}+l_{2}=l+1$ then $x_{1}=\sin ^{2}\left(\frac{\left(l_{1}-1 / 2\right) \pi}{l}\right)=$ $\sin ^{2}\left(\frac{\left(-l_{1}+l+1 / 2\right) \pi}{l}\right)=\sin ^{2}\left(\frac{\left(l_{2}-1 / 2\right) \pi}{l}\right)=x_{2}$, so that $P=0$.

(iii) The first equality in (4.4.5) can be shown by considering the action of a subgroup $\tilde{W}$ of the Weyl group $W$ of $s o(2 k+1)$ on $Q^{k}:=\left\{h=\left(l_{1}, \ldots l_{k}\right): l_{j}=\right.$ $1, \ldots, l\}$. The subgroup $\tilde{W}$ has order $k ! 2^{k-1}$, and it is generated by the maps $R_{s, t}$ and $P_{s, t}$ given as follows: For $t<s \leq k$, the reflection in the root $L_{s}+L_{t}$ acts on $Q^{k}$ by the map $R_{s, t}: h \mapsto\left(l_{1}, \ldots, l+1-l_{s}, \ldots, l+1-l_{t}, \ldots, l_{k}\right)$, and the reflection on the root $L_{t}-L_{s}$ by the map $P_{s, t}: h \mapsto\left(l_{1}, \ldots, l_{s}, \ldots l_{t}, \ldots, l_{k}\right)$. We omit the verification that these maps do satisfy the relations of the Weyl group generators. The action of $\tilde{W}$ on $Q^{k}$ preserves the products $P$, and furthermore, $T_{3}$ is a fundamental domain for $Q^{k}$ (see below). We provide the verification that $T_{3}$ is a fundamental domain for the action of $\tilde{W}$ on $Q^{k}$. The proof is essentially the same as the proof that the Weyl chamber is a fundamental domain for the action of the Weyl group on the weight space; see for example [Hu], page 52. For $\sigma \in \tilde{W}$, write $T_{\sigma}$ for $\sigma\left(T_{3}\right)$.

(a) $Q^{k}=\bigcup_{\sigma \in \tilde{W}} T_{\sigma}:$ If $\vec{v} \in Q^{k}$, we want to show that there exist $h \in T_{3}$ and $\sigma \in \tilde{W}$ such that $\sigma(h)=\vec{v}$. First, take a permutation $\rho_{1} \in S_{k}$ such that $\vec{v}^{(1)}=\rho_{1}(\vec{v}) \in T$, i.e., the coordinates of $\vec{v}^{(1)}$ are in decreasing order. Proceed now by induction on $v_{1}^{(1)}+v_{2}^{(1)}+\cdots+v_{k}^{(1)}$ : If $v_{1}^{(1)}+v_{2}^{(1)} \leq l+1$, there is nothing to do. 
Otherwise, consider $\vec{u}^{(1)}=P_{1,2}\left(\vec{v}^{(1)}\right)=\left(l+1-v_{2}^{(1)}, l+1-v_{1}^{(1)}, v_{3}^{(1)}, \ldots, v_{k}^{(1)}\right)$ and take $\rho_{2} \in S_{k}$ such that $\vec{v}^{(2)}=\rho_{2}\left(\vec{u}^{(1)}\right) \in T$. Note that $v_{1}^{(1)}+v_{2}^{(1)}+\cdots+v_{k}^{(1)}>$ $v_{1}^{(2)}+v_{2}^{(2)}+\cdots+v_{k}^{(2)}$, so that the result follows by induction.

(b) We show that if $\vec{v} \in T_{\sigma} \cap T_{\rho}$ for distinct $\sigma, \rho \in \tilde{W}$, then $P_{\vec{v}}=0$ : For this it suffices to show that for $\gamma \in \tilde{W} \backslash\{1\}, T_{\sigma} \cap T_{\rho} \subseteq \partial T_{3}:=\left\{h \in T_{3}: l_{1}+l_{2}=\right.$ $l+1$ or $l_{i}=l_{i+1}$ for some $\left.i\right\}$. So suppose that $\alpha$ and $\beta$ are elements in $T_{3}$, that $\gamma$ is a non-trivial element of $\tilde{W}$, and that $\gamma(\alpha)=\beta$. The subgroup $\tilde{W}$ consists of signed permutations with an even number of -1 's. Hence, the tranformation $\gamma$ of $Q^{k}$ acts by first changing an even number of coordinates $\alpha_{i}$ to $l+1-\alpha_{i}$, and then by permuting the coordinates. So, there is a permutation $\pi \in S_{k}$ such that for each $i$, $\beta_{i}=\alpha_{\pi(i)}$ or $\beta_{i}=l+1-\alpha_{\pi(i)}$. Suppose that $\gamma$ is not an element of the permutation subgroup of $W$ (generated by the $P_{s, t}$ 's). Then, there are two indices $s<t$ such that $\beta_{i}=l+1-\alpha_{\pi(i)}$ for $i=s$, t. Since $\alpha_{\pi(s)}+\alpha_{\pi(t)} \leq \alpha_{1}+\alpha_{2} \leq l+1$, it follows that $\beta_{s}+\beta_{t} \geq l+1$. But also $\beta_{s}+\beta_{t} \leq \beta_{1}+\beta_{2} \leq l+1$. Therefore, $\beta_{1}+\beta_{2}=l+1$ and $\beta \in \partial T_{3}$. Otherwise, $\gamma$ is a non-trivial element of the permutation subgroup of $W$. But then, as $\beta$ is obtained by permuting coordinates of $\alpha$ and both vectors have decreasing coordinates, $\beta$ must have some repeated coordinates, so again $\beta$ lies in the boundary of $T_{3}$.

The second equality in (4.4.5) follows directly from the fact that permuting the entries of $h$ does not change the products $P$.

Consider again $X \in M_{k}(\mathbb{C})$ to be the Vandermonde matrix in the variables $\left(x_{i}\right)_{1 \leq i \leq k}$ with $x_{i}$ as in (4.4.2) and $Y \in M_{k}(\mathbb{C})$ to be the diagonal matrix with the eigenvalues $\left(x_{i}\right)_{1 \leq i \leq k}$. Then, the determinant of $Y X^{2}$ is given by $\operatorname{det}\left(Y X^{2}\right)=$ $\prod_{s=1}^{k} x_{s} \prod_{1 \leq s<t \leq k}\left(x_{s}-x_{t}\right)^{2}$. Hence, rewriting (4.4.3), by Lemma 4.4.2 we have

$$
Q=\frac{\sum_{l_{1}, \ldots, l_{k}=1}^{l} \operatorname{det}\left(Y X^{2}\right)}{2^{k-2} k ! \prod_{s=1}^{k} \sin ^{2}\left(\left(s+\frac{1}{2}\right) \pi / l\right) \prod_{1 \leq s<t \leq k} \sin ^{2}((s+t+1) \pi / l) \sin ^{2}((t-s) \pi / l)} .
$$

Lemma 4.4.3.

$$
\sum_{l_{1}, \ldots, l_{k}=1}^{l} \operatorname{det}\left(Y X^{2}\right)=\frac{l^{k} k !}{2^{2 k^{2}-k}}
$$

Proof. By the binomial formula, $x_{s}^{j}=\frac{1}{(2 i)^{2 j}} \sum_{r=0}^{2 j}\left(\begin{array}{c}2 j \\ r\end{array}\right)(-1)^{r} e^{i \alpha_{s} 2(r-j) \frac{\pi}{l}}$, and so we have $\sum_{l_{s}=1}^{l} x_{s}^{j}=\frac{1}{2^{2 j}}\left(\begin{array}{c}2 j \\ j\end{array}\right) l$. By definition and Lemma $2.1(i i)$,

$$
\begin{aligned}
& \sum_{l_{1}, \ldots, l_{k}=1}^{l}\left(\operatorname{det} X^{2}\right)(\operatorname{det} Y) \\
& =\sum_{l_{1}, \ldots, l_{k}=1}^{l} \sum_{\sigma, \rho \in S_{k}} s g(\sigma) s g(\rho) \prod_{t=1}^{k} x_{t}^{\left(\rho^{-1}(t)+\sigma^{-1}(t)-1\right)} \\
& =l^{k} \sum_{\sigma, \rho \in S_{k}} s g(\sigma) s g(\rho) \prod_{t=1}^{k} \frac{1}{2^{2\left(\rho^{-1}(t)+\sigma^{-1}(t)-1\right)}}\left(\begin{array}{c}
2\left(\rho^{-1}(t)+\sigma^{-1}(t)-1\right) \\
\left(\rho^{-1}(t)+\sigma^{-1}(t)-1\right)
\end{array}\right) \\
& =\frac{l^{k}}{2^{2 k^{2}}} \sum_{\sigma, \omega \in S_{k}} s g(\omega) \prod_{t=1}^{k}\left(\begin{array}{c}
2(t+\omega(t)-1) \\
(t+\omega(t)-1)
\end{array}\right)=\frac{l^{k} k !}{2^{2 k^{2}-k}} .
\end{aligned}
$$


By (4.3.5), (4.4.6), Lemma 4.4.3 and [W-1, Theorem 1.5, (iii)] we have proved

Theorem 4.4.4. (i) If $C \subseteq D$ is the pair of $\mathrm{II}_{1}$ factors defined at the beginning of section 4.4 , then its index $[D: C]$ is given by

$$
\frac{x^{2 m} l^{k}}{2^{2 k^{2}-1}} \frac{1}{\prod_{s=1}^{k} \sin ^{2}\left(\left(s+\frac{1}{2}\right) \pi / l\right) \prod_{1 \leq s<t \leq k} \sin ^{2}((s+t+1) \pi / l) \sin ^{2}((t-s) \pi / l)},
$$

where $x$ is as in (4.3.1).

(ii) For each diagram $\lambda \in \Gamma_{m}\left(q^{2 k}, q\right)$ there exists a subfactor of the hyperfinite $\mathrm{II}_{1}$ factor with trivial relative commutant and index $\left[D_{\lambda}: C_{\lambda}\right]$ given by

$$
\frac{x^{2 m} l^{k}}{2^{2 k^{2}-1}} \frac{\prod_{s=1}^{k} \sin ^{2}\left(\left(l_{s}-\frac{1}{2}\right) \pi / l\right) \prod_{1 \leq s<t \leq k} \sin ^{2}\left(\left(l_{s}+l_{t}-1\right) \pi / l\right) \sin ^{2}\left(\left(l_{s}-l_{t}\right) \pi / l\right)}{\prod_{s=1}^{k} \sin ^{4}\left(\left(s+\frac{1}{2}\right) \pi / l\right) \prod_{1 \leq s<t \leq k} \sin ^{4}((t+s+1) \pi / l) \sin ^{4}((t-s) \pi / l)},
$$

where $l_{s}=\lambda_{s}+k-s+1$

If $\lambda$ is such that $\lambda_{1}^{\prime}>k$, consider $\tilde{\lambda}$ as defined in Lemma 4.4.1.

4.5 Index for subfactors of type C: The symplectic case $S p(2 k)$. This is the case where the values for $r$ and $q$ are given by $q=e^{\pi i / l}$ and $r=q^{-2 k-1}$, with $2 k+1<l, k$ positive and $l$ even. We have, by [W-2, Theorem $6.4,(\mathrm{c})]$,

$$
\Gamma\left(q^{-2 k-1}, q\right)=\left\{\lambda: \lambda_{1} \leq k \text { and } \lambda_{1}^{\prime} \leq \frac{l}{2}-k-1\right\} .
$$

For $\lambda \in \Gamma\left(q^{-2 k-1}, q\right)$ define $\lambda^{\prime}$, the transpose of $\lambda$, by $\left(\lambda^{\prime}\right)_{i}=\lambda_{i}^{\prime}$, so that $\left(\lambda_{1}^{\prime}\right)^{\prime}=$ $\lambda_{1} \leq k$. Furthermore, we have that $Q_{\lambda}\left(q^{-2 k-1}, q\right)=\chi^{\left(\lambda^{\prime}\right)}(D(q))$, where $D(q) \in$ $S p(2 k) \cap U(2 k)$ is the diagonal matrix with eigenvalues $\left\{q^{ \pm 2 i}, i=1, \ldots ., k\right\}$, by (4.3.4). The character formula for the representation of $S p(2 k)$ given by $\lambda=$ $\left(\lambda_{1}, \ldots, \lambda_{k}\right)$ is the following (see [Mi], [FH, Ch. 24], or [We]):

$$
\chi^{(\lambda)}(D(q))=\tilde{\chi}^{(\lambda)}(D(q))=\frac{\operatorname{det}\left(\left(q^{2 s l_{j}}-q^{-2 s l_{j}}\right)_{s, j}\right)}{\operatorname{det}\left(\left(q^{2 s l_{j}^{0}}-q^{-2 s l_{j}^{0}}\right)_{s, j}\right)},
$$

where $l_{j}:=\lambda_{j}+k-j+1, l_{j}^{0}:=k-j+1$ and $\tilde{\chi}^{(\lambda)}$ is the character of the restriction of $\lambda$ to $U(2 k)$. This formula can be written as

$$
\chi^{(\lambda)}(D(q))=\prod_{s=1}^{k} \frac{\left[2 l_{s}\right]_{q}}{\left[2 l_{s}^{0}\right]_{q}} \prod_{1 \leq s<t \leq k} \frac{\left[l_{s}+l_{t}\right]_{q}\left[l_{s}-l_{t}\right]_{q}}{\left[l_{s}^{0}+l_{t}^{0}\right]_{q}\left[l_{s}^{0}-l_{t}^{0}\right]_{q}}
$$

By (4.3.5) we need to compute $Q:=\sum_{\lambda \in \Gamma\left(q^{-2 k-1}, q\right)} Q_{\lambda}^{2}\left(q^{-2 k-1}, q\right)$. By (4.5.2),

$$
\begin{aligned}
Q & =\sum_{\left\{\lambda: \lambda^{\prime} \in \Gamma\left(q^{-2 k-1}, q\right)\right\}}\left(\chi^{(\lambda)}(D(q))\right)^{2} \\
& =\frac{\sum_{\left\{\lambda: \lambda^{\prime} \in \Gamma\left(q^{-2 k-1}, q\right)\right\}} \prod_{s=1}^{k}\left[2 l_{s}\right]_{q}^{2} \prod_{1 \leq s<t \leq k}\left[l_{s}+l_{t}\right]_{q}^{2}\left[l_{s}-l_{t}\right]_{q}^{2}}{\prod_{s=1}^{k}\left[2 l_{s}^{0}\right]_{q}^{2} \prod_{1 \leq s<t \leq k}\left[l_{s}^{0}+l_{t}^{0}\right]_{q}^{2}\left[l_{s}^{0}-l_{t}^{0}\right]_{q}^{2}} \\
& =\frac{\sum_{\left\{\lambda: \lambda^{\prime} \in \Gamma\left(q^{-2 k-1}, q\right)\right\}} \prod_{s=1}^{k} y_{s} \prod_{1 \leq s<t \leq k}\left(x_{s}-x_{t}\right)^{2}}{\prod_{s=1}^{k} \sin ^{2}(2 s \pi / l) \prod_{1 \leq s<t \leq k} \sin ^{2}((t+s) \pi / l) \sin ^{2}((t-s) \pi / l)},
\end{aligned}
$$


where we use the same trigonometrical identity as in section 4.4 , and where

$$
x_{s}=\sin ^{2}\left(l_{s} \pi / l\right) \text { and } y_{s}=\sin ^{2}\left(2 l_{s} \pi / l\right) .
$$

Lemma 4.5.1. (i) Set $T_{1}=\left\{\lambda: \lambda^{\prime} \in \Gamma\left(q^{-2 k-1}, q\right)\right\}=\left\{\lambda: \lambda_{1}^{\prime} \leq k\right.$ and $\lambda_{1} \leq$ $\left.\frac{l}{2}-k-1\right\}$. The map $\phi: T_{1} \rightarrow T_{2}:=\left\{\left(l_{1}, \ldots, l_{k}\right): \frac{l}{2}>l_{1}>\ldots .>l_{k}>0\right\}$ is a bijection, where $\lambda \mapsto \lambda+(k, k-1, \ldots, 1)$.

(ii) Set $P=\prod_{s=1}^{k} y_{s} \prod_{1 \leq s<t \leq k}\left(x_{s}-x_{t}\right)^{2}$. Then

$$
\sum_{\lambda \in T_{1}} P=\sum_{h \in T_{2}} P=\sum_{h \in T} P
$$

where $T=\left\{h=\left(l_{1}, \ldots, l_{k}\right): \frac{l}{2} \geq l_{1} \geq \ldots \geq l_{k} \geq 1\right\}$.

$$
\sum_{h \in T} P=\frac{1}{k !} \sum_{l_{1}, \ldots, l_{k}=1}^{\frac{l}{2}} P=\frac{1}{2^{k} k !} \sum_{l_{1}, \ldots, l_{k}=1}^{l} P .
$$

Proof. (i) Evident. (ii) For the second equality in (4.5.5), if $h=\left(l_{1}, \ldots, l_{k}\right)$ has $l_{i}=l_{j}$ for $i \neq j$ then $P=0$. If $l_{1}=\frac{l}{2}$ the expression is also 0 . (iii) For the first equality in (4.5.6), permuting the entries of $h \in T$ does not change the products. For the second equality in (4.5.6), since $x_{j}=\sin ^{2}\left(\frac{l_{j} \pi}{l}\right)=\sin ^{2}\left(\frac{\left(l-l_{j}\right) \pi}{l}\right)$ and $y_{j}=\sin ^{2}\left(2 l_{j} \pi / l\right)=\sin ^{2}\left(2\left(l-l_{j}\right) \pi / l\right)$, the products do not change if we repla๔e any $l_{j}$ by $l-l_{j}$.

Remark. As in the orthogonal cases, (iii) can be shown by considering the action of the Weyl group $W$ for $s p(2 k)$ on $Q^{k}:=\left\{h=\left(l_{1}, \ldots, l_{k}\right): l_{i}=1, \ldots, l\right\}$. The Weyl group $W$ has order $2^{k} k$ !; its action on $Q^{k}$ preserves the products $P$, and finally, $T$ is the fundamental domain for $Q^{k}$, in the same sense as in sections 4.4 and 4.6.)

Consider again $X \in M_{k}(\mathbb{C})$ to be the Vandermonde matrix in the variables $\left(x_{i}\right)_{1 \leq i \leq k}$ with $x_{i}$ as in (4.5.4), and $Y \in M_{k}(\mathbb{C})$ to be the diagonal matrix with the eigenvalues $\left(y_{i}\right)_{1 \leq i \leq k}$. Then, the determinant of $Y X^{2}$ is given by $\operatorname{det}\left(Y X^{2}\right)=$ $\prod_{s=1}^{k} y_{s} \prod_{1 \leq s<t \leq k}\left(x_{s}-x_{t}\right)^{2}$. Rewriting (4.5.3) and using Lemma 4.5.1, we see that

$$
Q=\frac{1}{2^{k} k !} \frac{\sum_{l_{1}, \ldots, l_{k}=1}^{l} \operatorname{det}\left(Y X^{2}\right)}{\prod_{s=1}^{k} \sin ^{2}(2 s \pi / l) \prod_{1 \leq s<t \leq k} \sin ^{2}((s+t) \pi / l) \sin ^{2}((t-s) \pi / l)} .
$$

\section{Lemma 4.5.2.}

$$
\sum_{l_{1}, \ldots, l_{k}=1}^{l} \operatorname{det}\left(Y X^{2}\right)=\frac{l^{k} k !}{2^{2 k^{2}-k}}
$$

Proof. By the binomial formula, $x_{s}^{j}=\frac{1}{(2 i)^{2 j}} \sum_{r=0}^{2 j}\left(\begin{array}{c}2 j \\ r\end{array}\right)(-1)^{r} e^{i l_{s} 2(r-j) \frac{\pi}{l}}$, and since $y_{s}=4\left(x_{s}-x_{s}^{2}\right)$, we have $\sum_{l_{s}=1}^{l} y_{s} x_{s}^{j}=\frac{1}{2^{2 j+1}}\left(\begin{array}{c}2(j+1) \\ j+1\end{array}\right) l \frac{1}{j+2}$. Then, by Lemma $2.1(i)$, 


$$
\begin{aligned}
& \sum_{l_{1}, \ldots, l_{k}=1}^{l}\left(\operatorname{det} X^{2}\right)(\operatorname{det} Y) \\
& =\sum_{\sigma, \rho \in S_{k}} s g(\sigma) \operatorname{sg}(\rho)\left\{\prod_{t=1}^{k} \frac{l}{2^{2\left(\rho^{-1}(t)+\sigma^{-1}(t)-2\right)+1}} \frac{1}{\left(\sigma^{-1}(t)+\rho^{-1}(t)\right)}\right. \\
& \left.\times\left(\begin{array}{c}
2\left(\rho^{-1}(t)+\sigma^{-1}(t)-1\right) \\
\left(\rho^{-1}(t)+\sigma^{-1}(t)-1\right)
\end{array}\right)\right\} \\
& =\frac{l^{k}}{2^{2 k^{2}-k}} \sum_{\sigma, \omega \in S_{k}} s g(\omega) \prod_{t=1}^{k} \frac{1}{(\omega(t)+t)}\left(\begin{array}{c}
2(t+\omega(t)-1) \\
(t+\omega(t)-1)
\end{array}\right)=\frac{l^{k} k !}{2^{2 k^{2}-k}} .
\end{aligned}
$$

By (4.3.5), (4.5.7), Lemma 4.5.2 and [W-1, Theorem 1.5, (iii)] we have proved

Theorem 4.5.3. (i) If $C \subseteq D$ is the pair of $\mathrm{II}_{1}$ factors defined at the beginning of section 4.5 , then its index $[D: C]$ is given by

$$
\frac{x^{2 m} l^{k}}{2^{2 k^{2}+1}} \frac{1}{\prod_{s=1}^{k} \sin ^{2}(2 s \pi / l) \prod_{1 \leq s<t \leq k} \sin ^{2}((s+t) \pi / l) \sin ^{2}((t-s) \pi / l)},
$$

where $x$ is as in (4.3.1).

(ii) For each diagram $\lambda \in \Gamma_{m}\left(q^{-2 k-1}, q\right)$ there exists a subfactor of the hyperfinite $\mathrm{II}_{1}$ factor with trivial relative commutant and index

$\left[D_{\lambda}: C_{\lambda}\right]=\frac{x^{2 m} l^{k}}{2^{2 k^{2}+1}} \frac{\prod_{s=1}^{k} \sin ^{2}\left(2 l_{s} \pi / l\right) \prod_{1 \leq s<t \leq k} \sin ^{2}\left(\left(l_{s}+l_{t}\right) \pi / l\right) \sin ^{2}\left(\left(l_{t}-l_{s}\right) \pi / l\right)}{\prod_{s=1}^{k} \sin ^{4}(2 s \pi / l) \prod_{1 \leq s<t \leq k} \sin ^{4}((t+s) \pi / l) \sin ^{4}((t-s) \pi / l)}$,

where $l_{s}=\lambda_{s}+k-s+1$.

4.6 Index for subfactors of type D: The even orthogonal case $O(2 k)$. This is the case where the values for $r$ and $q$ are given by $q=e^{\pi i / l}$ and $r=q^{2 k-1}$, with $2<2 k-1<l, l>3$. We have by [W-2, Theorem 6.4, (a)]

$\Gamma\left(q^{2 k-1}, q\right)=\left\{\lambda: \lambda_{1}+\lambda_{2} \leq l-2 k+2\right.$ and $\left.\lambda_{1}^{\prime}+\lambda_{2}^{\prime} \leq 2 k\right\} \cup\left\{\left[l-2 k+2,1^{2 k-2}\right]\right\}$.

Lemma 4.6.1. If $\lambda \in \Gamma\left(q^{2 k-1}, q\right)$, and $\lambda_{1}^{\prime}>k$, then there exists $\psi(\lambda)=\tilde{\lambda} \in$ $\Gamma\left(q^{2 k-1}, q\right)$ such that $\tilde{\lambda}_{1}^{\prime} \leq k-1$ and such that $Q_{\lambda}\left(q^{2 k-1}, q\right)=Q_{\tilde{\lambda}}\left(q^{2 k-1}, q\right)$. Furthermore, the map $\psi$ is a bijection from $\Gamma\left(q^{2 k-1}, q\right) \cap\left\{\lambda: \lambda_{1}^{\prime}>k\right\}$ onto $\Gamma\left(q^{2 k-1}, q\right) \cap\left\{\lambda: \lambda_{1}^{\prime} \leq k-1\right\}$.

Proof. For $\lambda \in \Gamma\left(q^{2 k-1}, q\right)$ with $\lambda_{1}^{\prime}>k$ define $\psi(\lambda)=\tilde{\lambda}$ by $\tilde{\lambda}_{i}^{\prime}=\lambda_{i}^{\prime}$ for $i=2, \ldots, \lambda_{1}$ and $\tilde{\lambda}_{1}^{\prime}=2 k-\lambda_{1}^{\prime}$. We have that $\lambda_{1}^{\prime}>k$ and $\lambda_{1}^{\prime}+\lambda_{2}^{\prime} \leq 2 k$ give $\tilde{\lambda}_{2}^{\prime} \leq \tilde{\lambda}_{1}^{\prime}$ (so that $\tilde{\lambda}$ is a Young diagram), $\tilde{\lambda}_{1}^{\prime}<k$ and $\tilde{\lambda}_{1}^{\prime}+\tilde{\lambda}_{2}^{\prime} \leq 2 k$. Also, $\tilde{\lambda}_{1}+\tilde{\lambda}_{2}=\lambda_{1}+\tilde{\lambda}_{2} \leq \lambda_{1}+\lambda_{2} \leq$ $l-2 k+2$. Therefore, $\tilde{\lambda} \in \Gamma\left(q^{2 k-1}, q\right)$ and $\tilde{\lambda}_{1}^{\prime} \leq k-1$. Similarly, the inverse map is well defined: For $\tilde{\lambda} \in \Gamma\left(q^{2 k-1}, q\right)$ with $\tilde{\lambda}_{1}^{\prime}<k$, take $\lambda$ with $\lambda_{i}^{\prime}=\tilde{\lambda}_{i}^{\prime}$ for $i=2, \ldots, \tilde{\lambda}_{1}$ and $\lambda_{1}^{\prime}=2 k-\tilde{\lambda}_{1}^{\prime}$. We have that $\lambda_{1}^{\prime} \geq \lambda_{2}^{\prime}$ and $\lambda_{1}^{\prime}>k$. If $\tilde{\lambda}$ is not just one row (that is, if $\tilde{\lambda}_{1}^{\prime}>1$ ), then $\lambda_{1}+\lambda_{2} \leq l-2 k+2$. If $\tilde{\lambda}_{1}^{\prime}=1$, then either $\lambda$ is the hook $\left[l-2 k+2,1^{2 k-2}\right]$ (when $\tilde{\lambda}=[l-2 k+2]$ ), or we have $\lambda_{1}+\lambda_{2} \leq l-2 k+2$ (when $\tilde{\lambda}=\left[\tilde{\lambda}_{1}\right]$ with $\left.\tilde{\lambda}_{1}<l-2 k+2\right)$. The fact that $Q_{\lambda}\left(q^{2 k-1}, q\right)=Q_{\tilde{\lambda}}\left(q^{2 k-1}, q\right)$ follows from (4.3.3). 
For $\lambda \in \Gamma\left(q^{2 k-1}, q\right)$ with $\lambda_{1}^{\prime} \leq k, Q_{\lambda}\left(q^{2 k-1}, q\right)=\chi^{(\lambda)}(D(q))$, and $D(q) \in O(2 k)$ is the diagonal matrix with eigenvalues $\left\{q^{ \pm 2(i-1)}, i=1, \ldots ., k\right\}$, where 1 appears twice, by (4.3.4). The character formula for the representation of $O(2 k)$ given by $\lambda=\left(\lambda_{1}, \ldots, \lambda_{k}\right)$ is the following (see for example [Mi, page 362] or [FH, page 410]):

If $\lambda_{k}=0$,

$$
\chi^{(\lambda)}(D(q))=\tilde{\chi}^{(\lambda)}(D(q))=\frac{\operatorname{det}\left(\left(q^{2(i-1) \alpha_{j}}+q^{-2(i-1) \alpha_{j}}\right)_{i, j}\right)}{\operatorname{det}\left(\left(q^{2(i-1) \alpha_{j}^{0}}+q^{-2(i-1) \alpha_{j}^{0}}\right)_{i, j}\right)} .
$$

If $\lambda_{k} \neq 0$,

$$
\begin{aligned}
\chi^{(\lambda)}(D(q)) & =\tilde{\chi}^{(\lambda)}(D(q))+\tilde{\chi}^{\left(\lambda_{-}\right)}(D(q)) \\
& =2 \frac{\operatorname{det}\left(\left(q^{2(i-1) \alpha_{j}}+q^{-2(i-1) \alpha_{j}}\right)_{i, j}\right)}{\operatorname{det}\left(\left(q^{2(i-1) \alpha_{j}^{0}}+q^{-2(i-1) \alpha_{j}^{0}}\right)_{i, j}\right)},
\end{aligned}
$$

where $\alpha_{j}=\lambda_{j}+k-j, \alpha_{j}^{0}=k-j, \lambda_{-}=\left(\lambda_{1}, \ldots, \lambda_{k-1},-\lambda_{k}\right)$, and $\tilde{\chi}^{(\lambda)}$ is the character of the restriction of $\lambda$ to $S O(2 k)$.

By the Weyl identity for the denominator of the character formula $\chi^{(\lambda)}(D(q))$, we can write

$$
\frac{\operatorname{det}\left(\left(q^{2(i-1) \alpha_{j}}+q^{-2(i-1) \alpha_{j}}\right)_{i, j}\right)}{\operatorname{det}\left(\left(q^{2(i-1) \alpha_{j}^{0}}+q^{-2(i-1) \alpha_{j}^{0}}\right)_{i, j}\right)}=\prod_{1 \leq s<t \leq k} \frac{\left[\alpha_{s}+\alpha_{t}\right]_{q}\left[\alpha_{s}-\alpha_{t}\right]_{q}}{\left[\alpha_{s}^{0}+\alpha_{t}^{0}\right]_{q}\left[\alpha_{s}^{0}-\alpha_{t}^{0}\right]_{q}} .
$$

Define

$$
x_{s}=\sin ^{2}\left(\frac{\alpha_{s} \pi}{l}\right) .
$$

By (4.3.5) we need to compute $Q:=\sum_{\lambda \in \Gamma\left(q^{2 k-1}, q\right)} Q_{\lambda}^{2}\left(q^{2 k-1}, q\right)$. By Lemma 4.6.1, (4.6.1), (4.6.2), (4.6.3) and the usual basic trigonometric identities we have

$$
\begin{aligned}
Q= & \sum_{\left\{\lambda \in \Gamma\left(q^{2 k-1}, q\right): \lambda_{1}^{\prime} \leq k\right\}} Q_{\lambda}^{2}\left(q^{2 k-1}, q\right)+\sum_{\left\{\lambda \in \Gamma\left(q^{2 k-1}, q\right): \lambda_{1}^{\prime}>k\right\}} Q_{\lambda}^{2}\left(q^{2 k-1}, q\right) \\
= & 2 \sum_{\left\{\lambda \in \Gamma\left(q^{2 k-1}, q\right): \lambda_{1}^{\prime}<k\right\}} Q_{\lambda}^{2}\left(q^{2 k-1}, q\right)+\sum_{\left\{\lambda \in \Gamma\left(q^{2 k-1}, q\right): \lambda_{1}^{\prime}=k\right\}} Q_{\lambda}^{2}\left(q^{2 k-1}, q\right) \\
= & \frac{2 \sum_{\left\{\lambda \in \Gamma\left(q^{2 k-1}, q\right): \lambda_{1}^{\prime}<k\right\}} \prod_{1 \leq s<t \leq k}\left(x_{s}-x_{t}\right)^{2}}{\prod_{1 \leq s<t \leq k} \sin ^{2}((s+t) \pi / l) \sin ^{2}((t-s) \pi / l)} \\
& +\frac{4 \sum_{\left\{\lambda \in \Gamma\left(q^{2 k-1}, q\right): \lambda_{1}^{\prime}=k\right\}} \prod_{1 \leq s<t \leq k}\left(x_{s}-x_{t}\right)^{2}}{\prod_{1 \leq s<t \leq k} \sin ^{2}((s+t) \pi / l) \sin ^{2}((t-s) \pi / l)} .
\end{aligned}
$$

Lemma 4.6.2. (i) Set $T_{1}=\left\{\lambda \in \Gamma\left(q^{2 k-1}, q\right): \lambda_{1}^{\prime}=k\right\}$. The map $\phi: T_{1} \rightarrow T_{2}:=$ $\left\{\alpha=\left(\alpha_{1}, \ldots, \alpha_{k}\right): \alpha_{1}+\alpha_{2} \leq l-1\right.$ and $\left.l-1>\alpha_{1}>\alpha_{2}>\ldots>\alpha_{k}>0\right\}$ is a bijection, where $\lambda \mapsto \alpha=\lambda+(k-1, k-2, \ldots, 1,0)$. Similarly, there is a bijection between the sets $S_{1}=\left\{\lambda \in \Gamma\left(q^{2 k-1}, q\right): \lambda_{1}^{\prime}<k\right\}$ and $S_{2}=\left\{\alpha=\left(\alpha_{1}, \ldots, \alpha_{k}\right)\right.$ : $\alpha_{1}+\alpha_{2} \leq l-1$ and $\left.l-1>\alpha_{1}>\alpha_{2}>\ldots>\alpha_{k-1}>\alpha_{k}=0\right\}$. 
(ii) Set $P=\prod_{1 \leq s<t \leq k}\left(x_{s}-x_{t}\right)^{2}$. Then

$$
\begin{aligned}
& \sum_{\lambda \in T_{1}} P=\sum_{\alpha \in T_{2}} P=\sum_{\alpha \in T_{3}} P, \\
& \sum_{\lambda \in S_{1}} P=\sum_{\alpha \in S_{2}} P=\sum_{\alpha \in S_{3}} P,
\end{aligned}
$$

where $T_{3}=\left\{\alpha=\left(\alpha_{1}, \ldots, \alpha_{k}\right): l-1 \geq \alpha_{1} \geq \ldots \geq \alpha_{k} \geq 1, \alpha_{1}+\alpha_{2} \leq l\right\}$ and where $S_{3}=\left\{\alpha=\left(\alpha_{1}, \ldots, \alpha_{k}\right): l-1 \geq \alpha_{1} \geq \ldots \geq \alpha_{k}=0, \alpha_{1}+\alpha_{2} \leq l\right\}$ (note that if $k=2$ then $\left.S_{3}=\left\{\alpha=\left(\alpha_{1}, \alpha_{2}\right): l-1 \geq \alpha_{1} \geq \alpha_{2}=0\right\}\right)$.

(iii)

$$
\begin{aligned}
& \sum_{\alpha \in T_{3}} P=\frac{1}{2^{k-1} k !} \sum_{\alpha_{1}, \ldots, \alpha_{k}=1}^{l-1} P=\frac{1}{2^{k-1}} \sum_{\alpha \in T} P \\
& \sum_{\alpha \in S_{3}} P=\frac{1}{2^{k-2}(k-1) !} \sum_{\alpha_{1}, \ldots, \alpha_{k-1}=0}^{l-1} P=\frac{1}{2^{k-2}} \sum_{\alpha \in S} P,
\end{aligned}
$$

where $T=\left\{\alpha=\left(\alpha_{1}, \ldots, \alpha_{k}\right): l-1 \geq \alpha_{1} \geq \ldots \geq \alpha_{k} \geq 1\right\}$ and where for $k>2$, $S=\left\{\alpha=\left(\alpha_{1}, \ldots, \alpha_{k}\right): l-1 \geq \alpha_{1} \geq \ldots \geq \alpha_{k}=0\right\}$.

(iv)

$$
\begin{aligned}
& 4 \sum_{\alpha \in T_{1}} P+2 \sum_{\alpha \in S_{1}} P=\frac{1}{2^{k-3}} \sum_{\alpha \in T} P+\frac{1}{2^{k-3}} \sum_{\alpha \in S} P \\
= & \frac{1}{2^{k-3}} \sum_{\left\{l-1 \geq \alpha_{1} \geq \cdots \geq \alpha_{k-1} \geq \alpha_{k} \geq 0\right\}} P=\frac{1}{k ! 2^{k-3}} \sum_{\alpha_{1}, \ldots, \alpha_{k}=0}^{l-1} P .
\end{aligned}
$$

Proof. (i) Evident.

(ii) For the second equality in (4.6.5) and (4.6.6), note that if $\alpha=\left(\alpha_{1}, \ldots, \alpha_{k}\right)$ has $\alpha_{i}=\alpha_{j}$ for $i \neq j$ then $P=0$. Also, if $\alpha_{1}+\alpha_{2}=l$ then $x_{1}=\sin ^{2}\left(\frac{\alpha_{1} \pi}{l}\right)=$ $\sin ^{2}\left(\frac{\left(l-\alpha_{2}\right) \pi}{l}\right)=\sin ^{2}\left(\frac{\alpha_{2} \pi}{l}\right)=x_{2}$, so that $P=0$.

(iii) This proof is very similar to the one for Lemma 4.4.2, (iii), except that in this case one considers the action of the whole Weyl group $W$ of $s o(2 k)$, which has order $k ! 2^{k-1}$, on $Q^{k}:=\left\{h=\left(l_{1}, \ldots l_{k}\right): l_{j}=1, \ldots, l-1\right\}$. To prove the first equality in (4.6.7) one can show that the action of $W$ on $Q^{k}$ preserves the products $P$ and also that $T_{3}$ is a fundamental region for the action of $W$ on $Q^{k}$, i.e., $Q^{k}=\bigcup_{\sigma \in W} T_{\sigma}$ and if $\vec{v} \in T_{\sigma} \cap T_{\rho}$ for distinct $\sigma, \rho \in W$, then $P_{\vec{v}}=0$. For proving the first equality in (4.6.8), one can use the same argument, considering the subgroup $W_{0}$ of $W$ generated by the $R_{s, t}$ 's and the $P_{s, t}$ 's with $t<s \leq k-1$ (defined as in the $s o(2 k+1)$ case), which has order $(k-1) ! 2^{k-2}$ and is isomorphic to the Weyl group of $s o(2 k-2)$. The second equalities in (4.6.7) and (4.6.8) follow directly from the fact that permuting the entries of $\alpha$ does not change the the products $\mathrm{P}$.

(iv) Evident.

As in section 4.2 for the type A case, $\prod_{1 \leq s<t \leq k}\left(x_{s}-x_{t}\right)^{2}$ is the formula for the determinant of the square of a Vandermonde matrix $X$ in the variables $\left(x_{s}\right)_{1 \leq s \leq k}$ 
with $x_{s}$ as in (4.6.3). Then, by Lemma 4.6.2 we can rewrite (4.6.4) as

$$
Q=\frac{1}{k ! 2^{k-3}} \frac{\sum_{\alpha_{1}, \ldots, \alpha_{k}=0}^{l-1}(\operatorname{det} X)^{2}}{\prod_{1 \leq s<t \leq k} \sin ^{2}((s+t) \pi / l) \sin ^{2}((t-s) \pi / l)} .
$$

\section{Lemma 4.6.3.}

$$
\sum_{\alpha_{1}, \ldots \alpha_{k}=0}^{l-1}(\operatorname{det} X)^{2}=\frac{l^{k} k !}{2^{2 k^{2}-3 k+1}} .
$$

Proof. By the binomial formula we have $x_{s}^{j}=\frac{1}{(2 i)^{2 j}} \sum_{r=0}^{2 j}\left(\begin{array}{c}2 j \\ r\end{array}\right)(-1)^{r} e^{i \alpha_{s} 2(r-j) \frac{\pi}{l}}$, so that $\sum_{\alpha_{s}=0}^{l-1} x_{s}^{j}=\frac{1}{2^{2 j}}\left(\begin{array}{c}2 j \\ j\end{array}\right) l$. Then, by Lemma $2.1($ iii $)$, and as in Lemma 4.4.3,

$$
\begin{aligned}
& \sum_{\alpha_{1}, \ldots, \alpha_{k}=0}^{l-1}(\operatorname{det} X)^{2} \\
= & \sum_{\alpha_{1}, \ldots, \alpha_{k}=0}^{l-1} \sum_{\sigma, \rho \in S_{k}} s g(\sigma) \operatorname{sg}(\rho) \prod_{t=1}^{k} x_{t}^{\left(\rho^{-1}(t)+\sigma^{-1}(t)-2\right)} \\
= & \frac{l^{k}}{2^{2\left(k^{2}-k\right)}} \sum_{\sigma, \omega \in S_{k}} s g(\omega) \prod_{t=1}^{k}\left(\begin{array}{c}
2(t+\omega(t)-2) \\
(t+\omega(t)-2)
\end{array}\right)=\frac{l^{k} k !}{2^{k^{2}-3 k+1}} .
\end{aligned}
$$

By (4.3.5), (4.6.9), Lemma 4.6.3 and [W-1, Theorem 1.5, (iii)], we have proved Theorem 4.6.4. (i) If $C \subseteq D$ is the pair of $\mathrm{II}_{1}$ factors defined at the beginning of section 4.6 , then its index $[D: C]$ is equal to

$$
\frac{x^{2 m} l^{k}}{2^{2 k^{2}-2 k-1}} \frac{1}{\prod_{1 \leq s<t \leq k} \sin ^{2}((s+t) \pi / l) \sin ^{2}((t-s) \pi / l)}
$$

where $x$ is as in (4.3.1).

(ii) For each diagram $\lambda \in \Gamma_{m}\left(q^{2 k-1}, q\right)$ there exists a subfactor of the hyperfinite $\mathrm{II}_{1}$ factor with trivial relative commutant and index $\left[D_{\lambda}: C_{\lambda}\right]$ given by:

If $\lambda_{k}>0$,

$$
\frac{x^{2 m} l^{k}}{2^{2 k^{2}-2 k-3}} \frac{\prod_{1 \leq s<t \leq k} \sin ^{2}\left(\left(\alpha_{s}+\alpha_{t}\right) \pi / l\right) \sin ^{2}\left(\left(\alpha_{s}-\alpha_{t}\right) \pi / l\right)}{\prod_{1 \leq s<t \leq k} \sin ^{4}((s+t) \pi / l) \sin ^{4}((t-s) \pi / l)} .
$$

If $\lambda_{k}=0$,

$$
\frac{x^{2 m} l^{k}}{2^{2 k^{2}-2 k-1}} \frac{\prod_{1 \leq s<t \leq k} \sin ^{2}\left(\left(\alpha_{s}+\alpha_{t}\right) \pi / l\right) \sin ^{2}\left(\left(\alpha_{s}-\alpha_{t}\right) \pi / l\right)}{\prod_{1 \leq s<t \leq k} \sin ^{4}((s+t) \pi / l) \sin ^{4}((t-s) \pi / l)},
$$

where $\alpha_{j}=\lambda_{j}+k-j$. If $\lambda$ is such that $\lambda_{1}^{\prime}>k$, consider $\tilde{\lambda}$ as defined in Lemma 4.6.1.

\section{REFERENCES}

[Bi] J. Birman, Braids, links and mapping class groups, Ann. Math. Studies, vol. 82, Princeton, NJ: Princeton Univ. Press., 1974. MR 51:11477

[BW] J. Birman, H. Wenzl, Braids, link polynomials and a new algebra, Trans. AMS 313 (1989), 249-273. MR 90g:57004

[Ch] M. Choda, Index for factors generated by Jones' two sided sequence of projections, Pacific J. Math. 139 (1989), 1-16. MR 91h:46107 
[D] V. Drinfeld, Quantum groups, Proceedings for the ICM, Berkeley (1986), 798-820. MR 89f: 17017

[FH] W. Fulton, J. Harris, Representation Theory. A First Course, vol. 129, Springer-Verlag, Graduate Texts in Mathematics, 1991. MR 93a:20069

[GHJ] F. Goodman, P. De la Harpe, V. Jones, Coxeter graphs and towers of algebras, vol. 14, Springer-Verlag, MSRI Publications, 1989. MR 91c:46082

[GW] F. Goodman, H. Wenzl, Littlewood-Richardson coefficients for the Hecke algebra at roots of unity, Adv. Math. 82 (1990), 24-45. MR 91i:20013

[Hu] J. Humphreys, Introduction to Lie Algebras and Representation Theory, Springer-Verlag, 1972. MR 48:2197

[J] V. Jones, Index for subfactors, Invent. Math. 72 (1983), 1-25. MR 84d:46097

[Ji] M. Jimbo, Quantum R-matrix for the generalized Toda system, Commun. Math. Phys. 102 (1986), 537-547. MR 87h:58086

[M] J. Murakami, The Kauffman polynomial of links and representation theory, Osaka J. of Math. 24 (1987), 745-758. MR 89c:57007

[Mi] W. Miller, Symmetry groups and their applications, Academic Press, 1972. MR 49:5032

[O] A. Ocneanu, Chirality of Operator Algebras, "Subfactors", Taniguchi Symposium on Operator Algebras, World Scient. (1994), 39-63. MR 96e:46082

[Po1] S. Popa, Orthogonal pairs of *-subalgebras of finite Von Neumann algebras, Journal of Operator Theory 9 (1983), 253-268. MR 84h:46077

[Po2] S. Popa, Classification of subfactors: the reduction to commuting squares, Invent. Math. 101 (1990), 19-43. MR 91h:46109

[W-1] H. Wenzl, Hecke algebras of type $A_{n}$ and subfactors, Invent. Math. 92 (1988), 349-383. MR 90b:46118

[W-2] H. Wenzl, Quantum groups and subfactors of Lie type B, C, and D, Comm. Math. Phys. 133 (1990), 383-433. MR 92k:17032

[W-3] H. Wenzl, Braids and invariants of 3-manifolds, Invent. Math. 114 (1993), 235-275. MR 94i:57021

[We] H. Weyl, The classical groups, their invariants and representations, Princeton Univ. Press, 2nd ed., 1953. MR 1:42c (1st ed.)

The Fields Institute, 222 College St., Toronto, Ontario M5T 3J1, Canada

E-mail address: jerlijma@fields.utoronto.ca 